\title{
CLASSIFICATION OF RATIONAL SURFACES OF DEGREE 11 AND SECTIONAL GENUS 11 IN P4
}

\author{
HANS-CHRISTIAN GRAF V. BOTHMER and KRISTIAN RANESTAD*
}

\begin{abstract}
We use the BGG-correspondence to show that there are at most three possible Hilbert functions for smooth rational surfaces of degree 11 and sectional genus 11. Surfaces with one of these Hilbert functions have been classified by Popescu. The classification for a second one is done in this paper. For the third Hilbert function the classification is still open.
\end{abstract}

\section{Introduction}

In the classification of smooth embedded varieties a natural start is to determine which Hilbert polynomials occur. Next one can classify the possible Hilbert functions. A bold aim is to determine the irreducible components of the Hilbert scheme representing smooth varieties and give a description of a general member in each component.

For space curves the first question was answered by Gruson and Peskine [10], while the second and third are only partially answered. For smooth surfaces in $\mathrm{P}^{4}$ there are even fewer results. The only general ones are the bounds of Ellingsrud and Peskine [6] that give asymptotic restrictions on the Hilbert polynomials that occur. Most work has concentrated on small invariants, and even here the results are only partial: The first question is only completely answered up to degree 10 , on the other hand in this case also the second and third question is completely answered, although not explicitly.

The techniques involved in the classification of surfaces with small invariants have developed considerably over time. The only common feature is that the combination of different approaches required often give the arguments a certain ad hoc flavor. While proving that certain components are nonempty, i.e. to give examples, can often be done transparently, it is the converse result, that a component is empty, that seems to require a combination of techniques.

\footnotetext{
* Supported by the Schwerpunktprogramm "Global Methods in Complex Geometry" of the Deutsche Forschungs Gemeinschaft and by the strategic university program Suprema of NFR (project 154077/420).

Received March 22, 2007; in revised form June 23, 2007.
} 
In this paper we use the Tate resolution of the ideal sheaf $\mathscr{I}:=\mathscr{I}_{S}$ of a smooth surface $S$ in $\mathrm{P}^{4}$ with Hilbert polynomial $P_{S}(n)=\frac{11}{2} n^{2}-\frac{9}{2} n+1$ to determine the Hilbert function of $S$. The main new idea is to study complexes on the Grassmannian of linear subspaces of $\mathrm{P}^{4}$, as introduced by [7], that are analogs of the Beilinson Monad. The degeneracy loci of the maps of these complexes define special linear subspaces in $\mathrm{P}^{4}$.

Special linear subspaces are those where the cohomology of the restricted ideal sheaf differs from the cohomology of a general restriction. Lines in the surface and lines that intersect the surface $S$ in a scheme of large length are special. A special plane intersects the surface $S$ in a curve, or in 11 points in special position. A special hyperplane intersects the surface in a space curve which lies on more surfaces of small degree than the general one. The geometry of special linear subspaces allows us to determine which maps can occur in the Tate resolution.

The main results and the organization of the paper is as follows: In Section 2 we recall the basic facts about the Tate resolution of $\mathscr{I}(n)$, its relation to the Beilinson Monad and the corresponding complexes on the Grassmannians as explained by Eisenbud and Schreyer [7]. When the intersection between the linear subspace and the surface is improper, then the ideal sheaf of the intersection is not the restriction of the ideal sheaf. The difference is made precise by the excess conormal sheaf. (cf. Fulton [8]). In Sections 3 and 4 we use this excess sheaf to study the restriction of the ideal of the surface to special lines and planes respectively. In the Section 5 we recall how the diagrams of generic initial ideals, as introduced by Green [11], can be applied to classify plane sections of $S$. Section 6 is then devoted to determining the Hilbert function of $S$. We show that only three different functions are possible. They differ from the Hilbert polynomial only in degrees $n=1, \ldots, 5$, where their values are $(5,15,35,70,116),(5,15,35,69,116)$ and $(5,15,35,69,115)$ respectively. Popescu showed that the first function occurs with three different irreducible families. In [16] v. Bothmer, Erdenberger and Ludwig give an example with the second function which was found by a random search over $F_{2}$. In Section 7 and 8 we show that all smooth surfaces with this second Hilbert function belong to the same irreducible and unirational family. This is Theorem 8.4. In Section 9 we a geometric construction of the surfaces in this family (Theorem 9.2).

The third Hilbert function also occurs for an irreducible family of surfaces, but we are not able to determine whether any of the surfaces belonging to that family are smooth. This reflects the nature of our methods. The exterior algebra methods we employ do not distinguish between smooth and singular irreducible surfaces. It is in combination with geometric arguments that we are sometimes able to make that distinction. On the other hand the constructed examples needed to eventually prove that a component is nonempty are of- 
ten so rigid that Bertini type theorems do not easily apply. Therefore, explicit examples of smooth surfaces are constructed using the computer algebra program Macaulay2 [13]. Scripts are provided and documented on our website [17]. These examples are constructed algebraically over $\mathbf{Z}$, and computed over a finite field, so by the openness condition of smoothness they are smooth over the rational numbers, and hence also over C. (cf. [4], Appendix A).

\section{Preliminaries}

Notation 2.1 .

$W \quad$ a vector space of dimension 5

$E=\bigwedge W^{*}$ the exterior algebra over its dual space, with grading given by $\operatorname{deg}\left(W^{*}\right)=-1$

$\mathrm{P}^{4}=\mathbf{P}(W) \quad$ the Grothendieck projectivisation of $W$

$\mathrm{G}_{l} \quad$ the Grassmannian of codim $l$ linear subspaces in $\mathrm{P}^{4}$

$\mathrm{F}_{l} \quad$ the Flag variety of points in codim $l$ linear subspaces of $\mathrm{P}^{4}$

In this paper we use the BGG-correspondence of Bernštel̆n, Gel'fand and Gel'fand [2] in an explicit version described by Eisenbud, Fløystad and Schreyer in [5]. For every sheaf $\mathscr{F}$ on $\mathrm{P}(W)$ one can construct a canonical exact complex $T(\mathscr{F})$ over the exterior algebra $E$. This complex is called the Tate resolution, see [5, Section 4] for the construction. The terms of the Tate resolution can be explicitly described:

Theorem 2.2 (Bernšteĭn, Gel'fand and Gel'fand; Eisenbud, Fløystad and Schreyer). If $\mathscr{F}$ is a coherent sheaf on $\mathrm{P}(W)$, then the e-th term of the Tate resolution is

$T(\mathscr{F})^{e}=\bigoplus_{j} \operatorname{Hom}_{K}\left(E, H^{j}(\mathscr{F}(e-j))\right) \cong \bigoplus_{j} H^{j}(\mathscr{F}(e-j)) \otimes E(j-e)$.

Proof. [5, Theorem 4.1].

Now consider the incidence correspondence

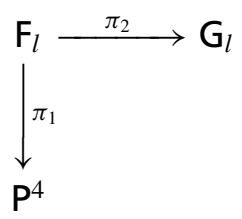

and the tautological sequence

$$
0 \longrightarrow \mathscr{U}_{l} \longrightarrow W \otimes \mathscr{O}_{\mathrm{G}_{l}} \longrightarrow \mathscr{Q}_{l} \longrightarrow 0 .
$$


In [7] Eisenbud and Schreyer define an additive functor $U_{l}$ from graded free modules over $E$ to locally free sheaves on $\mathrm{G}_{l}$ by taking $U_{l}(E(p))=\bigwedge^{p} \mathscr{U}_{l}$ and sending a map $\eta: E(q) \rightarrow E(q-p)$ to the map $U_{l}(\eta): \bigwedge^{q} \mathscr{U}_{l} \rightarrow \bigwedge^{q-p} \mathscr{U}_{l}$ defined by the element of $\wedge^{p} W^{*}$ corresponding to $\eta$ [7, Proposition 1.1]. We write $U_{l}(\mathscr{F})$ for $U_{l}(T(\mathscr{F}))$.

Theorem 2.3 (Beǐlinson; Eisenbud and Schreyer). If $\mathscr{F}$ is a sheaf on $\mathrm{P}^{n}$ then

$$
U_{l}(\mathscr{F})=R \pi_{2 *}\left(\pi_{1}^{*} \mathscr{F}\right)
$$

in the derived category.

Proof. [7, Theorem 1.2].

Remark 2.4. Notice that $U_{4}(\mathscr{F})$ is the Beillinson-Monad [1]. In this case $\mathscr{F}$ appears as the homology in step 0 . For $l \geq \operatorname{dim} \operatorname{supp} \mathscr{F}$ one can recover $\mathscr{F}$ from $U_{l}(\mathscr{F})$. [7, Proposition 1.3] In this paper we also use the partial information contained in $U_{l}(\mathscr{F})$ for $l \leq \operatorname{dim} \operatorname{supp} \mathscr{F}$.

We now consider the twisted ideal sheaf $\mathscr{I}_{S}(n)$ of a smooth surface in $\mathrm{P}^{4}$ and want to determine its Tate resolution $T\left(\mathscr{I}_{S}(n)\right)$. Its terms

$$
T\left(\mathscr{I}_{S}(n)\right)^{e}=\sum_{j=0}^{4} H^{j}\left(\mathscr{I}_{S}(n+e-j)\right) \otimes E(j-e)
$$

are given by Theorem 2.2. To determine its maps we apply the functor $U_{l}$ and use the geometry of $S$ to analyze the complexes $U_{l}(\mathscr{F})$. For fixed $l$ we use the notation

$$
F^{e}:=U_{l}(\mathscr{F})^{e}=\sum_{j=0}^{4} H^{j}\left(\mathscr{I}_{S}(n+e-j)\right) \otimes \bigwedge^{j-e} \mathcal{U}_{l} .
$$

Notice that only the terms $F^{-l} \ldots F^{4}$ are nonzero, so the complex $U_{l}(\mathscr{F})$ reduces to

$$
0 \rightarrow F^{-l} \stackrel{\psi^{-l+1}}{\longrightarrow} F^{-l+1} \stackrel{\psi^{-l+2}}{\longrightarrow} \ldots \stackrel{\psi^{3}}{\longrightarrow} F^{3} \stackrel{\psi^{4}}{\longrightarrow} F^{4} \longrightarrow 0 .
$$

Notice furthermore that each cohomology group $H^{i}\left(\mathscr{I}_{S}(k)\right)$ appears in at most one $F^{e}$.

In the following tables the entry $F^{e}$ in the row of $h^{j}$ and column $k$ indicates that $H^{j}\left(\mathscr{I}_{S}(k)\right) \otimes \bigwedge^{n-k} \mathscr{U}_{l}$ is a summand of $F^{e}$. For convenience we indicate $\wedge^{n-k} \mathscr{U}_{l}$ in the first row. 
For hyperplanes $(l=1)$ :

For planes $(l=2)$ :

\begin{tabular}{c|c|c|} 
& $\mathcal{O}(-1)$ & $\mathcal{O}$ \\
\hline$h^{4}$ & $F^{3}$ & $F^{4}$ \\
\hline$h^{3}$ & $F^{2}$ & $F^{3}$ \\
\hline$h^{2}$ & $F^{1}$ & $F^{2}$ \\
\hline$h^{1}$ & $F^{0}$ & $F^{1}$ \\
\hline$h^{0}$ & $F^{-1}$ & $F^{0}$ \\
\hline & $n-1$ & $n$
\end{tabular}

\begin{tabular}{c|c|c|c|} 
& $\mathcal{O}(-1)$ & $\mathscr{U}_{2}$ & $\mathcal{O}$ \\
\hline$h^{4}$ & $F^{2}$ & $F^{3}$ & $F^{4}$ \\
\hline$h^{3}$ & $F^{1}$ & $F^{2}$ & $F^{3}$ \\
\hline$h^{2}$ & $F^{0}$ & $F^{1}$ & $F^{2}$ \\
\hline$h^{1}$ & $F^{-1}$ & $F^{0}$ & $F^{1}$ \\
\hline$h^{0}$ & $F^{-2}$ & $F^{-1}$ & $F^{0}$ \\
\hline & $n-2$ & $n-1$ & $n$
\end{tabular}

For lines $(l=3)$ :

For points $(l=4)$ :

\begin{tabular}{c|c|c|c|c|} 
& $\mathcal{O}(-1)$ & $\bigwedge^{2} \mathscr{U}_{3}$ & $\mathscr{U}_{3}$ & $\mathcal{O}$ \\
\hline$h^{4}$ & $F^{1}$ & $F^{2}$ & $F^{3}$ & $F^{4}$ \\
\hline$h^{3}$ & $F^{0}$ & $F^{1}$ & $F^{2}$ & $F^{3}$ \\
\hline$h^{2}$ & $F^{-1}$ & $F^{0}$ & $F^{1}$ & $F^{2}$ \\
\hline$h^{1}$ & $F^{-2}$ & $F^{-1}$ & $F^{0}$ & $F^{1}$ \\
\hline$h^{0}$ & $F^{-3}$ & $F^{-2}$ & $F^{-1}$ & $F^{0}$ \\
\hline & $n-3$ & $n-2$ & $n-1$ & $n$
\end{tabular}

\begin{tabular}{c|c|c|c|c|c|} 
& $\mathcal{O}(-1)$ & $\wedge^{3} \mathscr{U}_{4}$ & $\wedge^{2} \mathscr{U}_{4}$ & $\mathscr{U}_{4}$ & $\mathcal{O}$ \\
\hline$h^{4}$ & $F^{0}$ & $F^{1}$ & $F^{2}$ & $F^{3}$ & $F^{4}$ \\
\hline$h^{3}$ & $F^{-1}$ & $F^{0}$ & $F^{1}$ & $F^{2}$ & $F^{3}$ \\
\hline$h^{2}$ & $F^{-2}$ & $F^{-1}$ & $F^{0}$ & $F^{1}$ & $F^{2}$ \\
\hline$h^{1}$ & $F^{-3}$ & $F^{-2}$ & $F^{-1}$ & $F^{0}$ & $F^{1}$ \\
\hline$h^{0}$ & $F^{-4}$ & $F^{-3}$ & $F^{-2}$ & $F^{-1}$ & $F^{0}$ \\
\hline & $n-4$ & $n-3$ & $n-2$ & $n-1$ & $n$
\end{tabular}

We mainly use this setup to calculate the cohomology of hyperplane, plane and line sections of $S$ via the following propositions:

Proposition 2.5. Let $L$ be a linear subspace of codimension $l$ in $\mathrm{P}^{4}$ and $\sigma \in \mathbf{G}_{l}$ the corresponding point in the Grassmannian. If $\psi^{i+1}=0$ then

$$
H^{i}\left(\left.\mathscr{I}_{S}(n)\right|_{L}\right)=\left.\operatorname{coker} \psi^{i}\right|_{\sigma} .
$$

In particular if $F^{i}=0$ then $H^{i}\left(\left.\mathscr{I}_{S}(n)\right|_{L}\right)=0$ for all linear subspaces $L \subset \mathrm{P}^{4}$.

Proof. By Theorem 2.3 we have a right exact sequence

$$
F^{i-1} \stackrel{\psi^{i}}{\longrightarrow} F^{i} \longrightarrow R^{i} \pi_{2 *}\left(\pi_{1}^{*} \mathscr{I}_{S}(n)\right) \longrightarrow 0 .
$$

Restricting to $\sigma$ we obtain

$$
\left.\left.\left.F^{i-1}\right|_{\sigma} \stackrel{\left.\psi^{i}\right|_{\sigma}}{\longrightarrow} F^{i}\right|_{\sigma} \longrightarrow H^{i}\left(\left.\mathscr{I}_{S}(n)\right|_{L}\right)\right) \longrightarrow 0 .
$$

REMARK 2.6. Notice that this proposition gives in a compact way the information one would get by repeatedly using the restriction sequence. 
We compare the restriction of the ideal sheaf to the ideal sheaf of the restriction:

Proposition 2.7. Let $\sigma \in \mathrm{G}_{l}$ be a linear subspace of codimension $l$, which does not intersect $S$ properly, but is not contained in $S$. Let $Z \subset S \cap \sigma$ the union of those components that are of codimension 1 in $\sigma$. Then there exists a locally free sheaf $J_{Z}$ on $Z$ such that

$$
\left.0 \longrightarrow J_{Z} \longrightarrow I_{S}\right|_{\sigma} \longrightarrow I_{S \cap \sigma} \longrightarrow 0 .
$$

Furthermore $J_{Z}$ completes the diagram

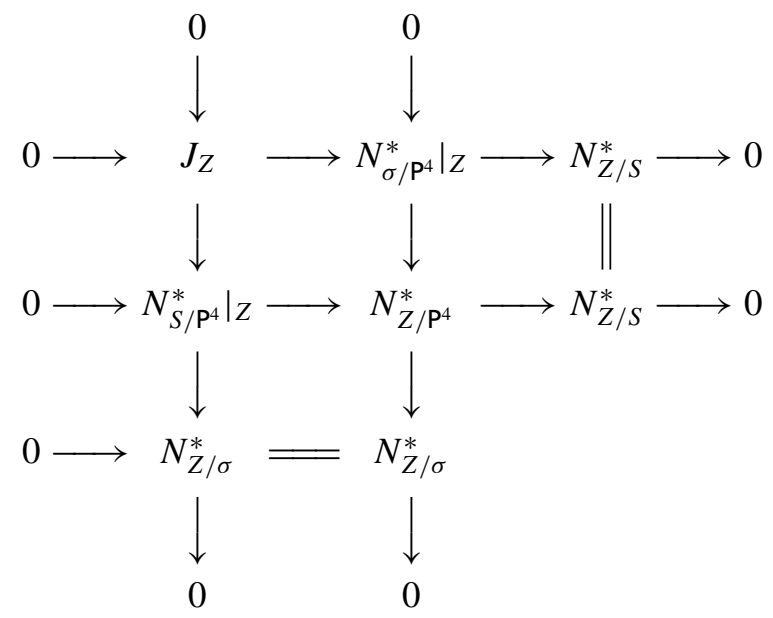

of conormal sheaves.

Proof. Locally at a point $z \in Z$, let $I=\mathscr{I}_{S, z}$ be the ideal of $S$ and $J=\mathscr{I}_{\sigma, z}$ the ideal of $\sigma$. Then the restriction $\left.I\right|_{\sigma}$ is given by $\frac{I}{I J}$, since it is the ideal $I$ tensored by the local coordinate ring $R_{\sigma}$ of $\sigma$ at $z$. The ideal of the intersection is $\frac{I+J}{J}$ inside $R_{\sigma}$. Furthermore there is a natural surjective map $\frac{I}{I J} \rightarrow \frac{I+J}{J}$ of $R_{\sigma}$ modules. The kernel is easily identified in the exact sequence:

$$
0 \longrightarrow \frac{I \cap J}{I J} \longrightarrow \frac{I}{I J} \longrightarrow \frac{I+J}{J} \longrightarrow 0 .
$$

Notice that the kernel is supported where the intersection is not proper, i.e. on $Z$. In particular there is a short exact sequence

$$
\left.0 \longrightarrow J_{Z} \longrightarrow \mathscr{I}_{S}\right|_{\sigma} \longrightarrow \mathscr{I}_{S \cap \sigma} \longrightarrow 0
$$


Now since $Z$ has pure codimension 1 in $\sigma$ it is a local complete intersection. Also $\sigma$ and $S$ are smooth, so we have an exact sequence

$$
0 \longrightarrow \frac{I \cap J}{I J} \longrightarrow \frac{I}{I^{2}} \otimes \frac{R_{\mathrm{P}^{4}, z}}{J} \longrightarrow \frac{J}{J^{2}} \longrightarrow 0 .
$$

This proves that $J_{Z}$ is locally free and fits into the proposed diagram.

REMARK 2.8. In Fulton's notation [9, Section 6.3], the dual of $J_{Z}$ is called the excess normal bundle of the fiber product

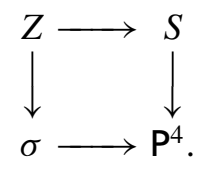

\section{Lines}

We consider multi-secants and lines in $S$.

Proposition 3.1. Let $L$ be a $k$-secant line to $S$. Then

$$
h^{1}\left(\left.\mathscr{I}_{S}(n)\right|_{L}\right)= \begin{cases}0 & \text { if } n>k-2 \\ k-n-1 & \text { if } n \leq k-2\end{cases}
$$

Proof. We are in the situation of Proposition 2.7 with $Z$ a scheme of length $k$ and an exact sequence

$$
\left.0 \longrightarrow J_{Z} \longrightarrow \mathscr{I}_{S}(n)\right|_{L} \longrightarrow \mathscr{I}_{Z}(n) \longrightarrow 0 \text {. }
$$

Since $J_{Z}$ has no $H^{1}$ we obtain $h^{1}\left(\left.\mathscr{I}_{S}(n)\right|_{L}\right)=h^{1}\left(\mathscr{I}_{Z}(n)\right)=h^{1}\left(\mathscr{O}_{\mathrm{P}^{1}}(n-k)\right)$.

Proposition 3.2. Let $L \subset S$ be a $(-k)$-line. Then

$$
\left.\mathscr{I}_{S}\right|_{L}=\mathscr{O}(-a) \oplus \mathscr{O}(-b)
$$

with $a+b=k+3$ and $0<a, b<k+3$.

Proof. If $L$ is contained in $S$, then $\left.\mathscr{I}_{S}\right|_{L}$ is the conormal bundle of $S$ restricted to $L$ and fits into the conormal bundle sequence

$$
\left.0 \longrightarrow N_{S / \mathrm{P}^{4}}^{*}\right|_{L} \longrightarrow N_{L / \mathrm{P}^{4}}^{*} \longrightarrow N_{L / S}^{*} \longrightarrow 0
$$

which reduces to

$$
\left.0 \longrightarrow N_{S / \mathrm{P}^{4}}^{*}\right|_{L} \longrightarrow 3 \mathscr{O}_{\mathrm{P}^{1}}(-1) \longrightarrow \mathcal{O}_{\mathrm{P}^{1}}(k) \longrightarrow 0
$$


from which the proposition follows.

Corollary 3.3. Let $L \subset S$ be $a(-k)$-line. If $n \geq k+1$ then

$$
h^{0}\left(\left.\mathscr{I}_{S}(n)\right|_{L}\right)-h^{0}\left(\mathscr{O}_{L}(n)\right)=n-k-2 .
$$

On the other hand, if $h^{1}\left(\left.\mathscr{I}_{S}(n)\right|_{L}\right) \neq 0$ then $k \geq n$.

Proof. $\left.\mathscr{I}_{S}(n)\right|_{L}=\mathscr{O}(n-a) \oplus \mathscr{O}(n-b)$ with $a, b<k+3$ by Proposition 3.2. For $n \geq k+1$ we have $h^{1}\left(\left.\mathscr{I}_{S}(n)\right|_{L}\right)=0$ and $h^{0}\left(\left.\mathscr{I}_{S}(n)\right|_{L}\right)=2 n-k-1$ since $a+b=k+3$.

Proposition 3.4. Let $L \subset \mathrm{P}^{4}$ be any line.

(1) If $h^{1}\left(\left.\mathscr{I}_{S}(n)\right|_{L}\right)=1$ then either $L$ is a $n+2$-secant line or $L \subset S$ and $L^{2} \leq-n$.

(2) If $h^{1}\left(\left.\mathscr{I}_{S}(n)\right|_{L}\right)=0$ and $h^{0}\left(\left.\mathscr{I}_{S}(n)\right|_{L}\right)-h^{0}\left(\mathscr{O}_{L}(n)\right)=1$ then $L \subset S$ with $L^{2}=3-n$.

Proof. From Proposition 3.1 and Corollary 3.3 we obtain (1). For $L \not \subset S$ one always has $h^{0}\left(\left.\mathscr{I}_{S}(n)\right|_{L}\right) \leq h^{0}\left(\mathscr{O}_{L}(n)\right)$, so claim (2) follows.

\section{Planes}

Throughout this section let $P \subset \mathrm{P}^{4}$ be a plane and $C \subset P \cap S$ be the 1dimensional component. By Proposition 2.7 we have the exact sequence

$$
\left.0 \longrightarrow J_{C} \longrightarrow N_{P / \mathbb{P}^{4}}^{*}\right|_{C} \longrightarrow N_{C / S}^{*} \longrightarrow 0
$$

which reduces to

$$
0 \longrightarrow J_{C} \longrightarrow 2 \mathscr{O}_{C}(-1) \longrightarrow \mathscr{O}_{C}(-C) \longrightarrow 0
$$

and shows $J_{C}=\mathscr{O}_{C}(C-2 H)$.

We can read off the degree of $C$ from the cohomology of either $I_{S \cap P}$ or $\left.I_{S}\right|_{P}$ :

Proposition 4.1. Let $P \subset \mathrm{P}^{4}$ be any plane and $C$ the curve component of $P \cap S$.

(1) If $n \geq-2$ then $h^{2}\left(\left.\mathscr{I}_{S}(n)\right|_{P}\right)=h^{2}\left(\mathscr{I}_{P \cap S}(n)\right)=\left(\begin{array}{c}\operatorname{deg} C-n-1 \\ 2\end{array}\right)$.

(2) If $h^{1}\left(\mathscr{O}_{C}(C+(n-2) H)\right)=0$ then $h^{1}\left(\left.\mathscr{I}_{S}(n)\right|_{P}\right)=h^{1}\left(\mathscr{I}_{P \cap S}(n)\right)$.

Proof. First we consider the cohomology of the short exact sequence

$$
\left.0 \longrightarrow \mathscr{O}_{C}(C+(n-2) H) \longrightarrow \mathscr{I}_{S}(n)\right|_{P} \longrightarrow \mathscr{I}_{S \cap P}(n) \longrightarrow 0 .
$$


Since $h^{2}\left(\mathscr{O}_{C}(C+(n-2) H)\right)=0$, we get $h^{2}\left(\left.\mathscr{I}_{S}(n)\right|_{P}\right)=h^{2}\left(\mathscr{I}_{P \cap S}(n)\right)$. In the second case $h^{1}\left(\mathscr{O}_{C}(C+(n-2) H)\right)=h^{2}\left(\mathscr{O}_{C}(C+(n-2) H)\right)=0$ and the second part of the proposition follows.

From the sequence

$$
0 \rightarrow \mathscr{I}_{P \cap S}(n) \rightarrow \mathscr{O}_{P}(n) \rightarrow \mathscr{O}_{P \cap S}(n) \rightarrow 0
$$

we obtain $h^{2}\left(\mathscr{I}_{P \cap S}\right)(n)=h^{1}\left(\mathscr{O}_{P \cap S}(n)\right)$ if $n \geq-2$. Furthermore

$$
h^{1}\left(\mathscr{O}_{P \cap S}(n)\right)=h^{1}\left(\mathscr{O}_{C}(n)\right)=h^{0}\left(\mathscr{O}_{C}(\operatorname{deg} C-3-n)\right)=\left(\begin{array}{c}
\operatorname{deg} C-n-1 \\
2
\end{array}\right)
$$

\section{Diagrams}

In the previous section we compared the restriction of the ideal of a surface in $\mathrm{P}^{4}$ to a plane with the ideal of the intersection of the surface and the plane. In this section we will concentrate on the latter. We recall from [11] how the different Hilbert functions of plane algebraic sets are read off from certain diagrams representing the generic initial ideals of their ideals.

Notation 5.1. Consider

$K[a, b, c]$ the coordinate ring of $\mathrm{P}^{2}$

gin $I$ the generic initial ideal of $I$ with respect to the reverse lexicographic order with $a<b<c$.

\section{ReMARK 5.2.}

(1) $I$ is saturated if and only if gin $I$ is saturated.

(2) if gin $I$ is saturated an $a^{i} b^{j} c^{k} \in \operatorname{gin} I$ then also $a^{i} b^{j} \in \operatorname{gin} I$.

(3) The Hilbert function and Hilbert polynomial of $I$ and gin $I$ are the same.

Definition 5.3. Let $I \subset K[a, b, c]$ be a saturated ideal. We represent the generic initial ideal gin $I$ by a diagram of $x$ 's and 0 's in $\mathrm{N}_{0} \times \mathrm{N}_{0}$. An $x$ in the point $(i, j)$ means that $a^{i} b^{j} \in \operatorname{gin} I$ and a 0 means $a^{i} b^{j} \notin$ gin $I$.

We also set

$$
\begin{aligned}
& d(I)=\min \left\{i \mid \exists a^{i} b^{j} \in \operatorname{gin} I\right\} \\
& e(I)=\#\left\{a^{i} b^{j} \notin \operatorname{gin} I \mid i \geq d\right\}
\end{aligned}
$$


EXAMPLE 5.4. gin $I=\left(a^{4}, a^{3} b, a^{2} b^{3}\right)$ is represented by

\begin{tabular}{c|cc|cccc} 
& $\vdots$ & $\vdots$ & & $\vdots$ & & \\
& 0 & 0 & $x$ & $x$ & $x$ & \\
& 0 & 0 & $x$ & $x$ & $x$ & \\
& 0 & 0 & 0 & $x$ & $x$ & $\ldots$ \\
& 0 & 0 & 0 & $x$ & $x$ & \\
& 0 & 0 & 0 & 0 & $x$ & \\
\hline & & & & $i$ & &
\end{tabular}

We have $d(I)=2$ and $e(I)=4$. Notice that $d(I)$ is the number of columns with only 0's and $e$ is the number of 0's outside of these columns.

REMARK 5.5. Since gin $I$ is an ideal we have 0's left and below each 0, and $x$ 's right and above of each $x$. Also generic initial ideals are Borel-fixed, i.e. for $i \geq 1$ we have

$$
a^{i} b^{j} \in \operatorname{gin} I \Longrightarrow a^{i+1} b^{i-1} \in \operatorname{gin} I .
$$

This means that we also have $x$ 's on the diagonal right and below of each $x$.

Proposition 5.6. Let $\mathscr{I}$ be an ideal sheaf on $\mathrm{P}^{2}$ and $I=\bigoplus H^{0}(\mathscr{I}(n))$ the corresponding saturated ideal. Let $H_{I}(n)=h^{0}\left(\mathscr{O}_{\mathrm{P}^{2}}(n)\right)-h^{0}(\mathscr{I}(n))$ be the Hilbert function of $V(I)$. Then

$H_{I}(n)=\#\{0$ 's below and on the diagonal $i+j=n$ in the diagram of gin $I\}$.

Proof. The monomials not contained in gin $I$ form a basis of $K[a, b, c] / I$.

Proposition 5.7. Let $I \subset K[a, b, c]$ be a saturated ideal. Then $d(I)$ as defined above is the degree of the curve components of $V(I)$.

Proof. For large $n$ the number of 0's on and below $i+j=n$ increases by $d(I)$ in each step, so the linear term of the Hilbert polynomial of $V(I)$ has coefficient $d(I)$.

Proposition 5.8. Let $I \subset K[a, b, c]$ be a saturated ideal. Then $e(I)$ as defined above is the degree of the dimension 0 component of $V(I)$.

Proof. The difference of the constant in the Hilbert polynomial of $V(I)$ and that of a plane curve of degree $d(I)$ is precisely $e$.

Useful for the geometric interpretation is the following 
Remark 5.9. It follows from a theorem of Ellia and Peskine [11, Theorem 4.4] that one can sometimes read off special positions of points in $V(I)$ according to the following rule:

If in the $i$ 'th column we have at least three $x$ 's to the right of three 0 's in column $i-1$, then there exists a curve of degree $i-d(I)$ passing through $n$ points of $V(I)$, where $n$ is the number of 0 's in columns $d(I)+1, \ldots, i-1$. The converse of this is not true in general.

Proposition 5.10. Let $\mathscr{I}$ be an ideal sheaf on $\mathrm{P}^{2}, I=\bigoplus H^{0}(\mathscr{I}(n))$ the corresponding saturated ideal and $r$ the number of 0 's with $i \geq d(I)$ lying above the diagonal $i+j=n$, then

$$
h^{1}(\mathscr{I}(n))=r
$$

Proof. The number $r$ is the difference between the Hilbert function and the Hilbert polynomial of $V(I)$ at $n$.

We again turn to our smooth surface $S$ of degree 11 in $\mathrm{P}^{4}$.

REMARK 5.11. Since the degree of the curve component of a plane section $S \cap P$ is bounded by the degree of $S$, it follows that for $h^{1}\left(\mathscr{I}_{S \cap P}(n)\right)=r$ there are only finitely many saturated generic initial ideals.

Example 5.12. Let $X \subset \mathrm{P}^{2}$ be a finite subscheme of degree 11 that is not contained in any conic section. For the diagram of $X$ this means that we must have no $x$ 's on and below the $i+j=2$ line, and eleven 0's altogether. The possible such diagrams are

$$
\left|\begin{array}{llll}
0 & x & x & x \\
0 & x & x & x \\
0 & x & x & x \\
0 & x & x & x \\
0 & x & x & x \\
0 & x & x & x \\
0 & 0 & x & x \\
0 & 0 & 0 & x
\end{array}\right| \begin{array}{llll}
0 & x & x & x \\
0 & x & x & x \\
0 & x & x & x \\
0 & x & x & x \\
0 & 0 & x & x \\
0 & 0 & x & x \\
0 & 0 & 0 & x \\
0
\end{array} \mid \begin{array}{llll}
0 & x & x & x \\
0 & x & x & x \\
0 & 0 & x & x \\
0 & 0 & x & x \\
0 & 0 & x & x \\
0 & 0 & 0 & x \\
\hline
\end{array}
$$

and

$\begin{array}{llll}0 & x & x & x \\
0 & x & x & x \\
0 & x & x & x \\
0 & 0 & x & x \\
0 & 0 & 0 & x \\
0 & 0 & 0 & x\end{array}\left|\begin{array}{llll}x & x & x & x \\
0 & x & x & x \\
0 & 0 & x & x \\
0 & 0 & x & x \\
0 & 0 & 0 & x \\
0 & 0 & 0 & x \\
0\end{array}\right|$\begin{tabular}{llll}
$x$ & $x$ & $x$ & $x$ \\
0 & $x$ & $x$ & $x$ \\
0 & $x$ & $x$ & $x$ \\
0 & 0 & $x$ & $x$ \\
0 & 0 & 0 & $x$ \\
0 & 0 & 0 & 0 \\
\hline
\end{tabular}


In the first case $X$ contains a subscheme of length 8 on a line, in the second case $X$ contains a subscheme of length 7 on a line, in the third case $X$ contains a subscheme of length 10 on a conic, in the fourth case $X$ contains a subscheme of length 6 on a line, in the first five cases $X$ is contained in a cubic, while in the last case $X$ is not contained in any cubic curve. Notice that each case is also distinguished by the corresponding values of $h^{1}\left(I_{X}(n)\right)$ for $n=3,4,5$. In fact, we get the following triples $(4,3,2),(3,2,1),(3,1,0),(2,1,0),(2,0,0)$ and $(1,0,0)$ respectively.

Example 5.13. Let $X \subset \mathrm{P}^{2}$ be the union of a quartic curve and a scheme of length 3. In the diagram of $X$ this means that the first four columns have all 0 's, and that there are three more 0 's. There are two possible diagrams:

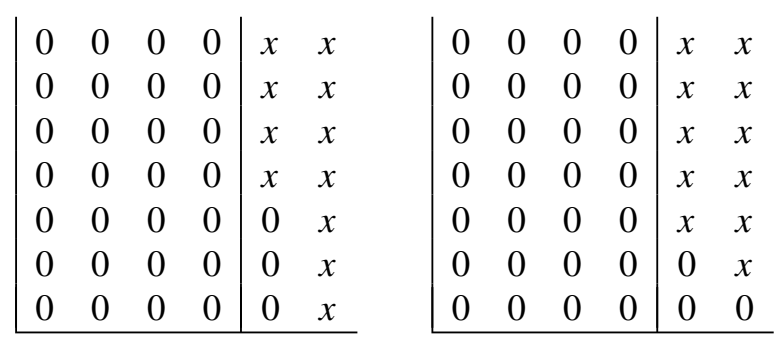

In both cases we have $h^{1}\left(I_{X}(4)\right)=3$. But only in the first case is the length 3 -subscheme on a line and only in this case is $h^{1}\left(I_{X}(6)\right)=1$.

\section{Rational Surfaces with $d=11, \pi=11$}

Let $S \subset \mathrm{P}^{4}$ be a rational surface of degree $d=11$ an section genus $\pi=11$. In this section we determine the possible Hilbert functions that $S$ can have and find restrictions on the maps in the Tate resolution. By Popescu [14], such surfaces have the following cohomology table for the ideal sheaf $\mathscr{I}_{S}$

\begin{tabular}{c|c|c|c|c|c|c|c|c|}
\hline$h^{4}$ & & & & & & & & \\
\hline$h^{3}$ & 11 & & & & & & & \\
\hline$h^{2}$ & & & 3 & 1 & & & & \\
\hline$h^{1}$ & & & & & 2 & $1+a$ & $b$ & $c$ \\
\hline$h^{0}$ & & & & & & $a$ & $10+b$ & $38+c$ \\
\hline & $\mathscr{I}_{S}(-1)$ & $\mathscr{I}_{S}(0)$ & $\mathscr{I}_{S}(1)$ & $\mathscr{I}_{S}(2)$ & $\mathscr{I}_{S}(3)$ & $\mathscr{I}_{S}(4)$ & $\mathscr{I}_{S}(5)$ & $\mathscr{I}_{S}(6)$
\end{tabular}

We now consider the Tate resolution of $\mathscr{I}_{S}(n)$. The most interesting part for 
our purposes is

$$
\begin{aligned}
& E(n-2) \\
& \oplus \quad(a+1) E(n-4)
\end{aligned}
$$

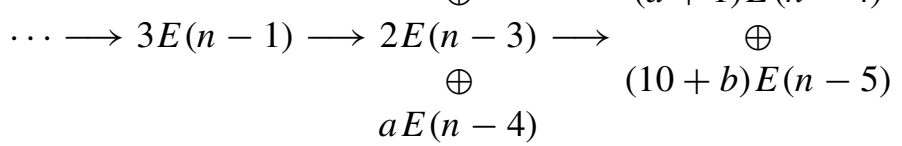

$$
\begin{aligned}
& \longrightarrow \begin{array}{c}
b E(n-5) \\
(38+c) E(n-6)
\end{array} \longrightarrow \cdots
\end{aligned}
$$

and in particular the subcomplex

$$
3 E(n-1) \stackrel{\alpha}{\longrightarrow} \underset{2 E(n-3)}{\oplus} \stackrel{E(n-2)}{\longrightarrow}(a+1) E(n-4) \stackrel{\gamma}{\longrightarrow} b E(n-5) .
$$

Applying the functor $U_{l}$ for $l=1,2,3$ we get complexes $U_{l}\left(\mathscr{I}_{S}(n)\right)$ with maps $U_{l}(\alpha), U_{l}(\beta)$ and $U_{l}(\gamma)$. By abuse of notation we often drop the functor. First we use Proposition 2.5 to compute the possible cohomology groups for the restriction of $\mathscr{I}_{S}(n)$ to linear subspaces:

Proposition 6.1. Let $\sigma \in \mathrm{G}_{l}$ be a linear subspace of codimension $l=$ $1,2,3$. Then the cohomology table of $\left.\mathscr{I}_{S}(n)\right|_{\sigma}$ for $n=2,3,4$ may have the following entries:

$$
\text { for } l=1 \text { (hyperplanes): }
$$

\begin{tabular}{|l|l|l|}
\hline & & \\
\hline & & \\
\hline $0 / 1$ & & \\
\hline $2 / 3$ & 3 & $a-1 / a / a+1$ \\
\hline & & $a / a+1 / a+2$ \\
\hline
\end{tabular}

for $l=2$ (planes):

\begin{tabular}{|c|c|c|}
\hline & & \\
\hline & & \\
\hline $0 / 1$ & & \\
\hline$?$ & $1 \ldots 4$ & $a-4 \ldots a+1$ \\
\hline & $?$ & $?$ \\
\hline
\end{tabular}

for $l=3$ (lines):

\begin{tabular}{|c|c|c|}
\hline & & \\
\hline & & \\
\hline 0 & & \\
\hline$?$ & $0 \ldots 5$ & $8-a \ldots a+1$ \\
\hline & $?$ & $?$ \\
\hline
\end{tabular}

where empty boxes stand for cohomologies that must be zero and question marks stand for cohomologies for which we have no restrictions so far. 
Proof. We use the first part of Proposition 2.5 repeatedly, and indicate the ranks of the vector bundles in the source and in the target to find the possible coranks of the maps. The cohomology group $H^{2}\left(\left.\mathscr{I}_{S}(2)\right|_{\sigma}\right)$ is the cokernel of $U_{l}(\alpha)$ whose source and target have ranks $3 \rightarrow 1,3 \cdot 2 \rightarrow 1$ and $3 \cdot 3 \rightarrow 1$ for $l=1,2,3$ respectively. In addition we must have $h^{2}\left(\left.\mathscr{I}_{S}(2)\right|_{\sigma}\right)=0$ for lines.

Similarly the cohomology groups $H^{1}\left(\left.\mathscr{I}_{S}(3)\right|_{\sigma}\right)$ are cokernels of $U_{l}(\alpha)$ whose source and target have ranks $0 \rightarrow 1+2,3 \rightarrow 1 \cdot 2+2$ and $3 \cdot 3 \rightarrow 1 \cdot 3+2$ respectively. Since the map $a \mathscr{O} \rightarrow(a+1) \mathcal{O}$ is always zero, the cohomology groups $H^{1}\left(\left.\mathscr{I}_{S}(4)\right|_{\sigma}\right)$ are cokernels of $U_{l}(\beta)$ whose source and target have ranks $2 \rightarrow 1+a, 1+2 \cdot 2 \rightarrow 1+a$ and $1 \cdot 3+2 \cdot 3 \rightarrow 1+a$ respectively.

For hyperplanes the intersection $S \cap \sigma$ is always a curve of degree 11 and arithmetic genus 11 . The possible cohomology dimensions $h^{1}\left(\left.\mathscr{I}_{S}(2)\right|_{\sigma}\right)$, $h^{0}\left(\left.\mathscr{I}_{S}(3)\right|_{\sigma}\right)$ and $h^{0}\left(\left.\mathscr{I}_{S}(4)\right|_{\sigma}\right)$ are therefore determined by Riemann-Roch.

The empty boxes of the proposition follow from the second part of Proposition 2.5.

Consider the linear part $\alpha_{1}$ of $\alpha$ in the Tate resolution. It is given by a $(3 \times 1)$ matrix with entries in $W^{*}$. These entries can be interpreted as points in $\mathrm{P}^{4}$.

Proposition 6.2. Let $\sigma$ be the linear space spanned by the entries of $\alpha_{1}$ in the Tate resolution of $\mathscr{I}$. Then $\sigma=P$ is a plane and $P \cap S$ contains the unique plane quintic curve on $S$.

Proof. If $\sigma=P$ is a plane, we consider the map

$$
3 \mathscr{U}_{2} \stackrel{\alpha_{1}}{\longrightarrow} \mathcal{O}
$$

on $\mathrm{G}_{2}$. It drops rank only on $P \in \mathrm{G}_{2}$. By Proposition 2.5 and 4.1 this happens if and only if $P \cap S$ contains a plane quintic.

If $\sigma$ is not a plane, we choose a line $L$ that contains $\sigma$ and consider the map

$$
3 \mathscr{U}_{3} \stackrel{\alpha_{1}}{\longrightarrow} \mathcal{O}
$$

on $\mathrm{G}_{3}$. If we restrict to $L$, this map vanishes and we obtain $h^{2}\left(\left.\mathscr{I}_{S}(2)\right|_{\sigma}\right)=1$ by Proposition 2.5. This is impossible on a line.

Let $C$ be the unique plane quintic curve on $S$, let $P$ be its span, and let $D=H-C$ be the residual curve to $C$ in a hyperplane section. Then $|D|$ is a pencil and $D^{2}$ is the length of the subscheme $R$ residual to $C$ in $P \cap S$.

LEMMA 6.3. $0 \leq D^{2} \leq 2$ and a general member of $|D|$ is a smooth curve of genus $g(D)=\bar{D}^{2}$.

Proof. Since $|D|$ has no fixed component $D^{2} \geq 0$. It remains to show that $D^{2} \leq 2$. 
Consider the short exact sequences of Proposition 2.7:

$$
\left.0 \longrightarrow J_{C}(3) \longrightarrow \mathscr{I}_{S}\right|_{P}(3) \longrightarrow \mathscr{I}_{S \cap P}(3) \longrightarrow 0 .
$$

and

$$
0 \longrightarrow J_{C}(3) \longrightarrow 2 \mathscr{O}_{C}(2) \longrightarrow \mathscr{O}_{C}(3 H-C) \longrightarrow 0 .
$$

Notice that $h^{0}\left(\mathscr{I}_{S \cap P}(3)\right)=0$, so taking cohomology in the former sequence yields $h^{1}\left(\left.\mathscr{I}_{S}\right|_{P}(3)\right)=h^{1}\left(J_{C}(3)\right)+h^{1}\left(\mathscr{I}_{S \cap P}(3)\right)$. Furthermore $h^{1}\left(\mathscr{I}_{S \cap P}(3)\right)=$ $h^{1}\left(\mathscr{I}_{R}(-2)\right)=D^{2}$. Therefore

$$
h^{1}\left(\left.\mathscr{I}_{S}\right|_{P}(3)\right)=h^{1}\left(J_{C}(3)\right)+h^{1}\left(\mathscr{I}_{S \cap P}(3)\right)=h^{1}\left(J_{C}(3)\right)+D^{2} .
$$

On the other hand $h^{1}\left(\left.\mathscr{I}_{S}\right|_{P}(3)\right) \leq 4$ by Proposition 6.1, so $h^{1}\left(J_{C}(3)\right)+D^{2} \leq 4$. First, this implies that $D^{2} \leq 4$, which means that $D \cdot C=(H-D) D=6-$ $D^{2} \geq 2$. But $\mathscr{O}_{C}(3 H-C)=\mathscr{O}_{C}(2 H+D)=\omega_{C}(D)$, so $h^{1}\left(\mathscr{O}_{C}(3 H-C)\right)=0$. Secondly, taking cohomology in the second sequence we get $h^{1}\left(J_{C}(3)\right) \geq$ $2 h^{1}\left(\mathscr{O}_{C}(2)\right)=2$, so $2+D^{2} \leq h^{1}\left(J_{C}(3)\right)+D^{2} \leq 4$, i.e. $D^{2} \leq 2$.

The pencil of curves $|D|$ has a base locus of length at most 2. By Bertini's Theorem the general member has singularities only in this base locus. But if the general $D$ is singular in the base locus, then $D^{2} \geq 4$, so we conclude that $D$ is smooth. Furthermore, $|D|$ is complete as a linear system, in fact $|H|$ is complete by Severi's Theorem and embeds $C$, so $D=H-C$ can only move in a pencil. But the general member of a complete pencil of curves on a rational surface, that does not have a fixed component, must be irreducible: In fact, the connected fibers of the Stein factorization of the map defined by $|D|$ are already linearly equivalent. Consequently, if the general element $D$ is a multiple of fibers, one could move one fiber while fixing the rest, contradicting the assumption that $|D|$ has no fixed component. Therefore the general member $D$ of the pencil $|D|$ is a smooth and irreducible curve of genus

$$
g(D)=\frac{1}{2}\left(D^{2}+D \cdot K\right)+1=\frac{1}{2}\left(D^{2}-2+D^{2}\right)+1=D^{2} .
$$

We take a closer look at the subcomplex

$$
3 E(n-1) \stackrel{\alpha}{\longrightarrow} \underset{2 E(n-3)}{\oplus} \stackrel{\beta}{\longrightarrow}(a+1) E(n-4) \stackrel{\gamma}{\longrightarrow} b E(n-5) .
$$

of the Tate resolution of $\mathscr{I}_{S}(n)$. The maps $\alpha, \beta$ and $\gamma$ can be given by matrices with entries of the following degrees:

$$
A=\left(\begin{array}{lll}
1 & 2 & 2 \\
1 & 2 & 2 \\
1 & 2 & 2
\end{array}\right) \quad B=\left(\begin{array}{ccc}
2 & \cdots & 2 \\
1 & \cdots & 1 \\
1 & \cdots & 1
\end{array}\right) \quad \Gamma=\left(\begin{array}{ccc}
1 & \cdots & 1 \\
\vdots & & \vdots \\
1 & \cdots & 1
\end{array}\right)
$$


Notice that $A, B$ and $\Gamma$ do not depend on the twist $n$. If $\sigma \in \mathrm{G}_{l}$ is a point in a Grassmannian, $L \subset \mathrm{P}^{4}$ the corresponding linear subspace of codimension $l$ and $M$ a matrix representing a morphism $\mu$ of graded free $E$ algebras, then we call

$$
\left.M\right|_{L}:=\left.\mathscr{U}_{l}(\mu)\right|_{\sigma}
$$

the restriction of $M$ to $L$. We will say that $M$ drops rank on $L$ if $\left.M\right|_{L}$ does not have maximal rank.

Proposition 6.4. Let $C_{i}(B)=\left(q, l_{1}, l_{2}\right)^{T}$ be a column of $B$. Then after coordinate changes and row operations we have one offollowing possibilities:

(1) $C_{i}(B)=\left(e_{3} \wedge e_{2}, e_{1}, e_{0}\right)^{T}$

(2) $C_{i}(B)=\left(0, e_{1}, e_{0}\right)^{T}$

(3) $C_{i}(B)=\left(e_{4} \wedge e_{3}+e_{2} \wedge e_{1}, e_{0}, 0\right)^{T}$

(4) $C_{i}(B)=\left(e_{2} \wedge e_{1}, e_{0}, 0\right)^{T}$

(5) $C_{i}(B)=\left(0, e_{0}, 0\right)^{T}$

(6) $C_{i}(B)=\left(e_{3} \wedge e_{2}+e_{1} \wedge e_{0}, 0,0\right)^{T}$

(7) $C_{i}(B)=\left(e_{1} \wedge e_{0}, 0,0\right)^{T}$

(8) $C_{i}(B)=(0,0,0)^{T}$

with $e_{0} \ldots e_{4}$ a basis of $V=W^{*}$.

Proof. We collect the coefficients of $q$ in a skew symmetric $5 \times 5$ matrix $M$. We say that $q$ has rank $r$ if $M$ has rank $2 r$.

If the linear forms are independent, we can assume that $q$ involves only the remaining 3 variables. Consequently we have rank $q \leq 1$. This gives the cases (1) and (2).

If the linear forms span a 1 dimensional space, we can assume that $q$ involves only the remaining 4 variables and $\operatorname{rank} q \leq 2$. This gives the cases (3), (4) and (5).

If both linear forms are zero, $q$ can involve all 5 variables and rank $q \leq 2$. This gives the last three cases

Proposition 6.5. Let $C_{i}(B)$ be a column of $B$. Then after row operations and coordinate changes one of the following holds

(1) $C_{i}(B)=\left(e_{3} \wedge e_{2}, e_{1}, e_{0}\right)^{T}$ and $P$ is contained in the $\mathrm{P}^{3}$ spanned by $e_{0}$, $e_{1}, e_{2}$ and $e_{3}$. Furthermore the line $L$ though $e_{0}$ and $e_{1}$ either intersects $S$ in a scheme of length at least 5 or $L \subset S$ with $L^{2} \leq-3$. If $L$ lies in $P$ then $C_{i}(B)$ vanishes on $P$.

(2) $C_{i}(B)=\left(e_{2} \wedge e_{1}, e_{0}, 0\right)^{T}$ and $P$ is spanned by $e_{0}, e_{1}$ and $e_{2}$. Furthermore each line $L$ that passes through $e_{0}$ and lies in $P$ either intersects $S$ in a scheme of length at least 6 or $L \subset S$ with $L^{2} \leq-4$. 
(3) $C_{i}(B)=\left(0, e_{1}, e_{0}\right)^{T}$ and the line $L$ though $e_{0}$ and $e_{1}$ either intersects $S$ in a scheme of length at least 6 or $L \subset S$ with $L^{2} \leq-4$.

Proof. First we look at the three possible cases and afterwards we exclude all other possibilities in Proposition 6.4. In both parts we use on the one hand the fact that $A B=0$ in the exterior algebra to obtain information about the matrix $A$ and on the other hand the geometric interpretation of $A$ and $B$ for various $l$.

The syzygy matrix of $C_{i}(B)=\left(e_{3} \wedge e_{2}, e_{1}, e_{0}\right)^{T}$ is

$$
\left(\begin{array}{ccccccc}
0 & 0 & 0 & e_{3} & e_{2} & -e_{1} & -e_{0} \\
0 & e_{1} & e_{0} & 0 & 0 & e_{3} \wedge e_{2} & 0 \\
e_{0} & 0 & e_{1} & 0 & 0 & 0 & e_{3} \wedge e_{2}
\end{array}\right)^{T}
$$

therefore the linear part of $A$ contains linear combinations of $e_{0} \ldots e_{3}$. This proves that $P$ lies in the $\mathrm{P}^{3}$ spanned by these points in $\mathrm{P}^{4}$. Since the line $L$ also lies in this $\mathrm{P}^{3}$ it is either contained in $P$ and $A$ drops rank on $L$ or it intersects $P$ in a point $\lambda e_{1}+\mu e_{0}$. On $L$ we then obtain

$$
\left.A\right|_{L}=\left(\begin{array}{ccc}
e_{3} \wedge e_{1} \wedge e_{0} & e_{2} \wedge e_{1} \wedge e_{0} & 0 \\
0 & 0 & \lambda e_{3} \wedge e_{2} \wedge e_{1} \wedge e_{0} \\
0 & 0 & \mu e_{3} \wedge e_{2} \wedge e_{1} \wedge e_{0}
\end{array}\right)^{T}
$$

which also has submaximal rank. This implies the geometric properties of (1) by Proposition 3.4. If $L$ lies in $P$ then $P=e_{0} \wedge e_{1} \wedge\left(\lambda e_{2}+\mu e_{3}\right)$ which annihilates all entries of $C_{i}(B)$.

The syzygy matrix of $C_{i}(B)=\left(e_{2} \wedge e_{1}, e_{0}, 0\right)^{T}$ is

$$
\left(\begin{array}{ccccc}
0 & 0 & e_{1} & e_{2} & -e_{0} \\
0 & e_{0} & 0 & 0 & e_{2} \wedge e_{1} \\
1 & 0 & 0 & 0 & 0
\end{array}\right)^{T}
$$

and $P$ is therefore spanned by $e_{2}, e_{1}$ and $e_{0}$. On the other hand $C_{i}(B)$ vanishes on all lines $L=\left(\lambda e_{1}+\mu e_{2}\right) \wedge e_{0}$ and consequently $B$ drops rank there. This implies (2) by Proposition 3.4.

If $C_{i}(B)=\left(0, e_{1}, e_{0}\right)^{T}$ this column vanishes on $L=e_{1} \wedge e_{0}$ and this implies (3).

Now we consider the other cases in Proposition 6.4.

The syzygy matrix $C_{i}(B)=\left(e_{4} \wedge e_{3}+e_{2} \wedge e_{1}, e_{0}, 0\right)^{T}$ is

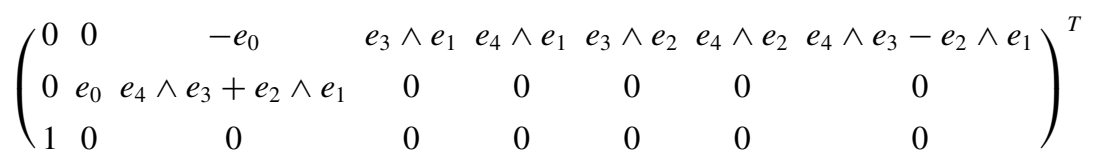

and therefore $P$ must be spanned by $e_{0}$ which is impossible. 
The column $C_{i}(B)=\left(0, e_{0}, 0\right)^{T}$ and $C_{i}(B)=(0,0,0)$ vanish on $e_{0}$ which is impossible since no sheaf on a point can have nonvanishing $H^{1}$.

The column $C_{i}(B)=\left(e_{3} \wedge e_{2}+e_{1} \wedge e_{0}, 0,0\right)^{T}$ has syzygy matrix

$$
\left(\begin{array}{ccccccc}
0 & 0 & e_{2} \wedge e_{0} & e_{3} \wedge e_{0} & e_{2} \wedge e_{1} & e_{3} \wedge e_{1} & e_{3} \wedge e_{2}-e_{1} \wedge e_{0} \\
0 & 1 & 0 & 0 & 0 & 0 & 0 \\
1 & 0 & 0 & 0 & 0 & 0 & 0
\end{array}\right)^{T}
$$

and there are no linear forms to span $P$.

Finally the syzygy matrix of $C_{i}(B)=\left(e_{1} \wedge e_{0}, 0,0\right)^{T}$ is

$$
\left(\begin{array}{cccc}
0 & 0 & e_{0} & e_{1} \\
0 & 1 & 0 & 0 \\
1 & 0 & 0 & 0
\end{array}\right)^{T}
$$

and $P$ must be spanned by $e_{0}$ and $e_{1}$ which is again impossible.

Remark 6.6. Since $B$ has corank 1 on any 6-secant there is a unique column of type (2) or (3) for each such line. Furthermore $B$ vanishes on a 7 -secant line.

Remark 6.7. Except for the observation that $A$ drops rank on $L$ in the first case, this classification was already obtained by Popescu in [14].

We now consider the case of several columns in the matrix $B$, and start by focusing on the linear part $B_{1}$ and the span of its entries $\mathrm{P}_{\text {span }} \subset \mathrm{P}^{4}$. We denote by $\mathrm{P}_{c}^{a}$ the column space and by $\mathrm{P}_{r}^{1}$ the row space of $B_{1}$. The Segre variety $\mathrm{P}_{r}^{1} \times \mathrm{P}_{c}^{a} \subset \mathrm{P}^{2 a+1}$ is described by a $2 \times(a+1)$ matrix and $B_{1}$ defines a birational map

$$
p: \mathrm{P}_{r}^{1} \times \mathrm{P}_{c}^{a} \longrightarrow \mathrm{P}_{\text {span }} \subset \mathrm{P}^{4}
$$

which can be interpreted as the projection from a linear space $\mathbf{P}_{\perp} \subset \mathrm{P}^{2 a+1}$ which is the space of linear relations between the entries of $B_{1}$. Denote by $T$ the image of $p$.

Lemma 6.8. Any quartic $X_{4}$ containing $S$ also contains $T$.

Proof. If a column of $B$ is of type (1) or (3) then the span $L$ of its linear entries is either contained in $S$ or at least a 5-secant to $S$ by Proposition 6.5. It is therefore contained in $X_{4}$. If a column of $B$ is of type (2) it has only one linear entry which represents a point of $S \subset X_{4}$ by Proposition 6.5 .

Corollary 6.9. $\operatorname{dim} T \leq 2$.

Proof. If $\operatorname{dim} T>2$ then by construction $a \geq 2$ and $S$ is contained in at least 2 independent quartics. Since these also contain $T$ by Lemma 6.8 we obtain a contradiction. 
LeMma 6.10. The intersection $Z=\mathrm{P}_{\perp} \cap \mathrm{P}_{r}^{1} \times \mathrm{P}_{c}^{a}$ is finite.

Proof. A point $(r, c) \subset \mathrm{P}_{r}^{1} \times \mathrm{P}_{c}^{a}$ is in $\mathrm{P}_{\perp}$ if and only if the entry of $B_{1}$ in the corresponding generalized row and column is zero. If $Z$ is infinite, one of the following happens

(1) a column of $B_{1}$ vanishes. This is impossible by Proposition 6.5

(2) several columns of $B_{1}$ have rank 1 . Each of them gives a residual point in $P$.

(a) If this point moves, we obtain infinitely many residual points in $P$ which is impossible.

(b) If this point does not move, we obtain at least two columns that span only a point. Denote by $B^{\prime}$ the corresponding two columns of $B$ and by $B_{1}^{\prime}$ their linear part. Since there can be no zero column in $B_{1}^{\prime}$ we obtain

$$
B_{1}^{\prime}=\left(\begin{array}{cc}
e_{1} & 0 \\
0 & e_{1}
\end{array}\right)
$$

after row and column operations. By Proposition 6.5 the point $e_{1}$ lies in $P$. If $P$ is spanned by $e_{1}, e_{2}$ and $e_{3}$ the same proposition shows that the 2-forms of $B^{\prime}$ are linear combinations of $e_{2} \wedge e_{3}$ and terms of the form $e_{1} \wedge *$. After eliminating the $e_{1}$-terms with column operations we obtain

$$
B^{\prime}=\left(\begin{array}{ccc}
\lambda e_{2} \wedge e_{3} & e_{1} & 0 \\
\mu e_{2} \wedge e_{3} & 0 & e_{1}
\end{array}\right)^{T}
$$

By Proposition 6.5 again the coefficients $\lambda$ and $\mu$ must be nonzero, but then we obtain the column $\left(0, \mu e_{1},-\lambda e_{1}\right)^{T}$ as a linear combination contradicting Proposition 6.5.

COROLlary 6.11. In the above notation:

(1) $a \leq \operatorname{dim} \mathrm{P}_{\text {span }}$

(2) $a \leq \operatorname{dim} T \leq a+1$

(3) $a \leq 2$.

Proof. If $\operatorname{dim} \mathrm{P}_{\text {span }}<a$ we have codim $\mathrm{P}_{\perp} \leq a$ and therefore

$$
\operatorname{dim} Z=\operatorname{dim}\left(\mathbf{P}_{\perp} \cap \mathbf{P}_{r}^{1} \times \mathbf{P}_{c}^{a}\right) \geq 1 .
$$

This contradicts Lemma 6.10. The variety $T$ is the image of the projection from $\mathrm{P}_{\perp}$ and $\mathrm{P}_{\perp} \cap \mathrm{P}_{r}^{1} \times \mathrm{P}_{c}^{a}$ is finite, so the fibers of the projection are at most 1-dimensional and the second part follows. The third part now follows from the second and Corollary 6.9 . 
We now turn to the case of $\operatorname{dim} \mathrm{P}_{\text {span }}=2$ and we denote by $C^{\prime}$ the dimension 1 component of $\mathrm{P}_{\text {span }} \cap S$. The $\mathrm{P}^{3}$ 's containing $\mathrm{P}_{\text {span }}$ generate a pencil of space curves $\left|D^{\prime}\right|$ residual to $C^{\prime}$. Notice that since plane curves in $S$ have degree at most 5 we have $\operatorname{deg} D^{\prime} \geq 6$.

Proposition 6.12. Let $D^{\prime} \subset \mathrm{P}^{3}$ be an irreducible space curve of degree at least 6 that is contained in no quadric but in a $a_{3}$-dimensional space of cubics, with $a_{3} \geq 3$. Then $D^{\prime}$ is either a septic of arithmetic genus 5 and $a_{3}=3$ or a sextic of arithmetic genus $a_{3}-1$ with $a_{3} \leq 4$.

Proof. Let $Z$ be the curve component of the intersection of cubics that contain $D^{\prime}$. Then $\operatorname{deg} Z \leq 7$ : Since $Z$ lies in the complete intersection of two cubics, $\operatorname{deg} Z^{\prime} \leq 9$. But degree 9 is impossible since such a curve only lies on two cubics. Degree 8 is also impossible since $Z$ would be linked $(3,3)$ to a line. Any curve linked $(3,3)$ to a line is contained in precisely a pencil of cubics. This latter result has a geometric version: If the line is reduced in the complete intersection, then it intersects the linked curve in a scheme of length 4. Thus by Bezout's Theorem, the line is contained in every cubic that contains the linked curve.

Since $D^{\prime} \subset Z$ is irreducible and the residual part $D^{\prime}-Z$ is at most a line, $Z$ is reduced. Now, by assumption there are at least a net of pencils of cubic surfaces that contain $Z$. So we consider the curve $E$ linked to $Z$ in a general such pencil. By Bertini's Theorem $E$ is singular only in the singular part of $Z$. Therefore $E$ must be reduced.

If $\operatorname{deg} Z=7$ then $E$ must be a plane conic or two skew lines. If $E$ is two skew lines, then at least one of them, say $L$, is not contained in $Z$. The union of $Z$ and the other line, say $L^{\prime}$, is linked $(3,3)$ to $L$. By the geometric property of linkage above, $L$ intersects $Z$ in a scheme of length 4 , so it must be contained in $Z$, contrary to the above. Therefore $E$ must be a plane conic and $Z$ has arithmetic genus 5 by the liaison formula. Furthermore, in this case $a_{3}=3$. In particular, if $a_{3}>3$, then $\operatorname{deg} D^{\prime} \leq \operatorname{deg} Z<7$. This proves our claim if $\operatorname{deg} D^{\prime}=7$.

If $\operatorname{deg} D^{\prime}=6, a_{3} \geq 3$ then either $D^{\prime}=Z$ or $D^{\prime}=Z+L$ where $L$ is an additional line. In the latter case $Z=D^{\prime} \cup L$ is reduced, and linked $(3,3)$ to a plane conic $E$ by the previous argument. In particular $D^{\prime} \cup L$ has arithmetic genus 5. If the line $L$ lies in the plane of $E$, then $D^{\prime}$ is linked $(3,3)$ to a plane cubic, so it is contained in a quadric, contrary to the assumption. If $L$ meets $M$ in a point, then $L \cup E$ has arithmetic genus 0 , and by liaison, $D^{\prime}$ has arithmetic genus 3 and lies in 4 cubics. If $L$ does not meet $E$, then $D^{\prime} \cup E$ is linked to the line $L$, so as above, $L$ intersects $D^{\prime}$ in a scheme of length 4 . Thus $D^{\prime}$ must have arithmetic genus 2 and $a_{3}=3$.

If, on the other hand, $D^{\prime}=Z$, then $E$ is a reduced curve of degree 3 . If 
$E$ is not connected, then it has a line component that must intersect $D^{\prime}$ in a scheme of length 4 as above. So this line would be contained in $Z$, contrary to our assumption. Therefore $E$ is connected. It is a plane cubic curve or a space curve of arithmetic genus 0 . In the first case $D^{\prime}$ would be linked $(3,3)$ to a plane cubic, and hence lie in a quadric hypersurface, against our assumption. In the second case, $D^{\prime}$ has arithmetic genus 3 and $a_{3}=4$.

Proposition 6.13. In the above notation, $a \leq 1$

Proof. If $a=2$ we have $\operatorname{dim} \mathrm{P}_{\text {span }} \geq 2$ by Corollary 6.11 .

If $\mathrm{P}_{\text {span }}$ is a plane, then we consider the pencil of residual space curves $\left|D^{\prime}\right|$ introduced above. As in the proof of Lemma 6.3 we may assume that the general member $D^{\prime}$ is a reduced and irreducible curve.

By Corollary 6.11(2) the projection $p$ is surjective. By Lemma 6.8 all $a+$ $2=4$ quartics in the ideal of $C^{\prime} \cup D^{\prime}$ also contain $T=\mathrm{P}_{\text {span }}$. By Proposition 6.1 the curve $C^{\prime} \cup D^{\prime}$ lies on no cubic. This implies that $D^{\prime}$ lies on 4 cubics and no quadric. Therefore $D^{\prime}$ has degree 6 and arithmetic genus 3 by Proposition 6.12 . In this case we must have $C^{\prime}=C, \mathrm{P}_{\text {span }}=P$ and $D^{\prime}=D$ since $S$ contains only one plane quintic by Proposition 6.2. But from Lemma 6.3 we know that $D$ is in fact smooth and irreducible of genus at most 2, so we get a contradiction.

If $\mathrm{P}_{\text {span }}=\mathrm{P}^{3}$, i.e. $\mathrm{P}_{\perp}=\mathrm{P}^{1}$ the projection $p$ is surjective: Every fiber is the intersection of a plane with $\mathrm{P}_{r}^{1} \times \mathrm{P}_{c}^{a}$ which contains at least 3 points, and these points could not lie in $\mathrm{P}_{\perp}$ since the Segre variety has no 3-secant lines. If $\mathrm{P}_{\text {span }}=\mathrm{P}^{4}$ and $\mathrm{P}_{\perp}$ is a point, then $T$ is a threefold since $\mathrm{P}_{r}^{1} \times \mathrm{P}_{c}^{a} \subset \mathrm{P}^{5}$ is not a cone. If $\mathrm{P}_{\perp}$ is outside of $\mathrm{P}_{c}^{a} \times \mathrm{P}_{r}^{1}$, we have $\operatorname{deg} T=3$ otherwise $\operatorname{deg} T=2$. In total $T$ is a threefold of degree at most 3 contradicting Lemma 6.8

From now on we may assume $a=0$ or 1 .

Proposition 6.14. If $a=0$, then $b=0$.

Proof. If $a=0$, then $B=\left(q, l_{1}, l_{2}\right)^{t}$ has to be one of the types in Proposition 6.5. None of them has linear exterior syzygies.

Proposition 6.15. If $a=1$ and $c=\left(l_{1}, l_{2}\right)^{t}$ is a column of $\Gamma$, then the linear forms $l_{1}$ and $l_{2}$ are independent.

Proof. If $c=(0,0)^{t}$, then the restriction of $c$ to any point $p$ vanishes, and $h^{1}\left(\left.\mathscr{I}_{S}(5)\right|_{p}\right) \geq 1$. This is impossible. If after coordinate changes $c=\left(e_{0}, 0\right)^{t}$, then the restriction to $e_{0}$ vanishes and we obtain a contradiction as before.

Proposition 6.16. If $a=1$ and $b \geq 1$ then $\mathrm{P}_{\text {span }}=\mathrm{P}^{1}$.

Proof. Since $B \Gamma=0$ the rows of $B$ have to be syzygies of the transpose of any column of $\Gamma$. The syzygies of $c^{t}=\left(l_{1}, l_{2}\right)$ with $l_{1}$ and $l_{2}$ independent 
are generated by

$$
\left(\begin{array}{lll}
l_{1} & 0 & l_{2} \\
0 & l_{2} & l_{1}
\end{array}\right) .
$$

So the linear forms in $B$ must all lie in the span of the $l_{i}$, i.e. $\mathrm{P}_{\text {span }} \subset\left\langle l_{1}, l_{e}\right\rangle$. Since by Corollary 6.11 the dimension of $\mathrm{P}_{\text {span }}$ is at least one in this case, the proposition follows.

Corollary 6.17. If $a=1$ then $b \leq 1$.

Proof. If $\mathrm{P}_{\text {span }} \neq \mathrm{P}^{1}$, then $b=0$. If $\mathrm{P}_{\text {span }}=\mathrm{P}^{1}$ we consider the linear part $B_{1}$ of $B$. Possible columns of $\Gamma$ must be among the syzygies of $B_{1}$. After coordinate changes there are only two possibilities for $B_{1}$ :

(1) If $B_{1}=\left(\begin{array}{cc}e_{0} & 0 \\ 0 & e_{1}\end{array}\right)$ then the syzygies of $B_{1}$ are generated by $\left(\begin{array}{cc}e_{0} & 0 \\ 0 & e_{1}\end{array}\right)$, so $b \leq 2$. If $b=2$ then $\Gamma=\left(\begin{array}{cc}e_{0} & 0 \\ 0 & e_{1}\end{array}\right)$ and some columns do not have full rank. This is impossible by Proposition 6.15.

(2) If $B_{1}=\left(\begin{array}{ll}e_{1} & 0 \\ e_{0} & e_{1}\end{array}\right)$ then the syzygies of $B_{1}$ are generated by $\left(\begin{array}{ll}e_{1} & 0 \\ e_{0} & e_{1}\end{array}\right)$. As before we obtain $b \leq 1$

COROLlaRY 6.18. The regularity of $\mathscr{I}_{S}$ is at most 7.

Proof. If $b=0$ then then the regularity of $\mathscr{I}_{S}$ is 6 . If $b=1$ then the regularity of $\mathscr{I}_{S}$ is 7 since $C=\left(l_{1}, l_{2}\right)^{t}$ does not have any linear syzygies, when the $l_{i}$ are linearly independent.

Recall the well known fact

LeMma 6.19. Let $E \subset \mathrm{P}^{3}$ be a non degenerate pure 1-dimensional scheme lying on a 3-dimensional set of quadrics. Then $E$ is defined by the $2 \times 2$ minors of a $2 \times 3$ matrix of linear form, i.e. a curve of degree 3 and arithmetic genus 0 .

Proof. Let $X$ be the scheme cut out by the 3 quadrics. First we prove that $X$ can not contain a surface. Assume it does, then this surface must be a plane $P^{\prime}$ and the residual scheme in $X$ is cut out by 3 independent linear forms. So $X$ is $P^{\prime}$ union a point. $E$ then must be contained in $P$ contradicting our assumptions.

So a general quadric in the ideal of $E$ is irreducible. The only curve on an irreducible quadric cut out by exactly 3 quadrics is a curve linked to a line in two quadrics, i.e. a cubic curve as described in the statement of the lemma. 
Proposition 6.20. $\operatorname{dim} \mathrm{P}_{\text {span }} \neq 2$.

Proof. Assume that $\mathrm{P}_{\text {span }}$ is a plane. Note that in this case only $a=1$ is possible. Let $C^{\prime}$ be the curve component of $\mathrm{P}_{\text {span }} \cap S$ and consider the pencil of residual space curves $\left|D^{\prime}\right|$ introduced above. Again, as in the proof of Lemma 6.3, we may assume that a general member $D^{\prime}$ is reduced and irreducible.

Since we consider $H:=\mathrm{P}^{3}$ containing $\mathrm{P}_{\text {span }}$, the matrix $B_{1}$ vanishes on $H$ and the ideal of $S \cap H=C^{\prime} \cup D^{\prime}$ contains a 3-dimensional space of quartics by Proposition 2.5 and no cubics by Proposition 6.1 .

The projection $p$ is surjective, since the preimage of a point is a line in $\mathrm{P}^{3}$ intersecting $\mathrm{P}_{r}^{1} \times \mathrm{P}_{c}^{1}$ in at least 2 points that can not both be contained in the projection center $\mathrm{P}_{\perp}=\mathrm{P}^{0}$. So $\mathrm{P}_{\text {span }}$ is contained in all 3 quartics of $I_{C^{\prime} \cup D^{\prime}}$ and therefore $D^{\prime}$ lies on three cubics and no quadrics. On the other hand, $S$ has no plane curves of degree more than 5 , so $\operatorname{deg} D^{\prime} \geq 6$.

Since $D^{\prime}$ is reduced and irreducible, Proposition 6.12 applies, and so either $D^{\prime}$ has degree 7 and arithmetic genus 5 , or $D^{\prime}$ has degree 6 and arithmetic genus 2 .

In the second case of $\operatorname{deg} C^{\prime}=11-6=5$ and we have $C^{\prime}=C, \mathrm{P}_{\text {span }}=P$ and $D^{\prime}=D$. By Lemma 6.3, the general curve $D$ is smooth and irreducible of genus $D^{2}=2$. In particular the intersection of $S$ with $P$ is the union of $C$ and a scheme of length 2 . Therefore the ideal of $S$ is not generated by sextics. Hence $b \geq 1$ and $\mathrm{P}_{\text {span }}=\mathrm{P}^{1}$ by Proposition 6.16.

This leaves us with the first case, where $D^{\prime}$ has degree 7 and arithmetic genus 5 and $\operatorname{deg} C^{\prime}=11-7=4$. In this case $\left(D^{\prime}\right)^{2}=3$, so the pencil of curves $\left|D^{\prime}\right|$ has a baselocus of length three in the plane $\mathrm{P}_{\text {span }}$. In addition $\mathrm{P}_{\text {span }}$ intersects $S$ in the quartic curve $C^{\prime}$, so if the baselocus of $\left|D^{\prime}\right|$ is disjoint from $C^{\prime}$, then we may conclude from Example 5.13 that $h^{1}\left(I_{\mathrm{P}_{\mathrm{span}} \cap S}(4)\right)=3$. Now $\left(C^{\prime}\right)^{2}=0$, so $h^{1}\left(\mathcal{O}_{C^{\prime}}\left(C^{\prime}+2 H\right)\right)=0$, therefore, by Proposition $4.1(2)$, we may compute the cohomology $h^{1}\left(\left.I_{S}(4)\right|_{\mathrm{P}_{\text {span }}}\right)=h^{1}\left(I_{\mathrm{P}_{\text {span }} \cap S}(4)\right)=3$. But by Proposition $6.1, h^{1}\left(\left.I_{S}(4)\right|_{\mathrm{P}_{\text {span }}}\right) \leq a+1=2$, which is in contradiction with the above. What remains is to show that the baselocus of $\left|D^{\prime}\right|$ is disjoint from $C^{\prime}$ in $\mathrm{P}_{\text {span }}$.

The proof is rather roundabout, and we start by considering again the projection $p$. There are two cases, either the projection center is contained in $\mathrm{P}_{r}^{1} \times \mathrm{P}_{c}^{1}$ or not. If not, then the pencil of lines given by the columns of $B_{1}$ does not have a basepoint. Since each of these line is at least a 5 secant line to $S$, the curve $C^{\prime}$ must be the plane quintic contrary to the above. If the projection center is contained in $\mathrm{P}_{r}^{1} \times \mathrm{P}_{c}^{1}$, then two lines are blown down by the projection. The image of one of them is a basepoint of the pencil given by the columns of $B_{1}$, say $e_{0}$. The other one corresponds to a column of $B_{1}$ whose entries only 
span a $\mathrm{P}^{0}$ say $e_{1}$. Therefore after a coordinate change we can assume

$$
B=\left(\begin{array}{cc}
q_{1} & q_{2} \\
e_{0} & 0 \\
e_{2} & e_{1}
\end{array}\right)
$$

Now consider the plane $P$ of the plane quintic. In the second column of $B$ we know by Proposition 6.5 (2) that $e_{1}$ lies in $P$. Furthermore, by this proposition, we know that $q_{2} \neq 0$. Therefore a general linear combination of columns must be of type (1) in Proposition 6.5. Without loss of generality we can assume this to be the first column.

By Proposition 6.5 (1) we know that the line through $e_{0}$ and $e_{2}$ lies in a $\mathrm{P}^{3}$ that also contains $P$. Therefore we have at least one point of $P$ on this line. This point can not be $e_{1}$ since $e_{0}, e_{1}$ and $e_{2}$ span $\mathrm{P}^{2}$. Therefore $P$ intersects $\mathrm{P}_{\text {span }}$ in at least two distinct points i.e. in a line. If $P=\mathrm{P}_{\text {span }}$ we are again in the case above. If $P \neq \mathrm{P}_{\text {span }}$ their span $H$ is a $\mathrm{P}^{3}$. The intersection curve $E=H \cap S$ then lies on 3 quartics and no cubics. The quartics contain $\mathrm{P}_{\text {span }}$ as argued above and $P$, since $P$ contains a plane quintic. Finally we have two possibilities; either the line of intersection $L=P \cap \mathrm{P}_{\text {span }}$ is contained in $S$, or it is not.

In the latter case, the residual curve $E-C-C^{\prime}$ has degree $\operatorname{deg}\left(E-C-C^{\prime}\right)=$ $11-5-4=2$ and must lie on 3 quadrics and no plane. This contradicts Lemma 6.19 .

LEMMA 6.21. If $L=P \cap \mathrm{P}_{\text {span }}$ is contained in $S$, then the baselocus of $\left|D^{\prime}\right|$ in $\mathrm{P}_{\text {span }}$ is disjoint from $C^{\prime}$.

Proof. The proof follows from a careful analysis of the reducible hyperplane section $E$.

Notice first that the second column of $B$ vanishes on $L$, so, by Proposition 6.5 (2), the selfintersection $L^{2} \leq-4$. The doubling of $L$ on $S$ is not contained in any of the planes $P$ or $\mathrm{P}_{\text {span }}$, since such a doubling would mean that $L^{2}=1$ on $S$. Therefore $A=C^{\prime}-L$ is a plane cubic with no component along $L$, and $B=C-L$ is a plane quartic curve with no component along $L$. The two curves $A$ and $B$ can only intersect on $L$, but if they do, then this intersection point would be a singular point on $S$, a contradiction. Therefore $A \cdot B=0$. On the other hand, $A \cdot L=3$ and $B \cdot L=4$, so the arithmetic genus $p(A+B+L)=p(A+L)+p(B)+(A+L) \cdot B-1=9$. The residual curve $G=E-A-B-L$ lies on 3 quadrics and in no plane, so by Lemma 6.19 is has degree 3 and arithmetic genus $p(G)=0$.

The union $G+B$ lies in the pencil $\left|D^{\prime}\right|=\left|H-C^{\prime}\right|$. Therefore $p\left(D^{\prime}\right)=$ $p(G+B)=p(G)+p(B)+G \cdot B-1=5$, while $p(E)=p(G)+p(A+B+$ 
$L)+G \cdot(A+B+L)-1=11$. Combined we get the intersection numbers $G \cdot B=3$ and $G \cdot(A+L)=0$.

If $G$ has a component along $L$, then $A+B+2 L$ is contained in the union of the two planes $P$ and $\mathrm{P}_{\text {span }}$, and $G-L$ has degree two and is contained in at least 4 quadrics. This means that $E$ is contained in 4 quartics, contrary to the above.

If $G$ has a component in common with $B$, then this component must have degree one or two. In the latter case, the residual part of $G$ would be a line that intersects the first component. But then it could not be a component of $A$, since $A$ and $B$ cannot intersect. So $0=(A+L) \cdot G \geq L \cdot G=2$, which is absurd.

The former case is similar if $G$ does not have a component in $A$. If $G$ has a component in $A$, then it must be a line $L_{A}$, that does not intersect the line component $L_{B}$ of $G$ in $B$. Thus $G=L_{A}+L_{0}+L_{B}$, and $3=G \cdot B=$ $\left(L_{0}+L_{B}\right) \cdot B \leq 1+L_{B} \cdot B$, so $L_{B} \cdot B \geq 2$. But $\left(L_{B}\right)^{2}=L_{B} \cdot B-L_{B} \cdot(B-$ $\left.L_{B}\right) \geq 2-3=-1$. On the other hand the intersection $\left(H-2 L_{B}\right) \cap L_{B}=$ $\left(A+L+\left(B-L_{B}\right)+L_{A}+L_{0}\right) \cap L_{B}=\left(L+\left(B-L_{B}\right)+L_{0}\right) \cap L_{B}$ is finite of length at least five, while the intersection number $\left(H-2 L_{B}\right) \cdot L_{B} \leq 3$, a contradiction.

Therefore $G$ has no component in $P$ and intersect $B$ and $P$ in a scheme of length 3. In particular $G$ does not intersect $L$, so $G$ has no component in common with $A$ and, since $A \cdot G=0$, does not intersect $A$. In conclusion $G$ intersect $\mathrm{P}_{\text {span }}$ in a scheme of length three outside $A \cup L$.

The lemma concludes the proof of the proposition.

Proposition 6.22. If $a=1$ and $\mathrm{P}_{\mathrm{span}}=L$ is a line, then $b=1$.

Proof. We treat the possible matrices $B_{1}$ case by case:

If $B_{1}=\left(\begin{array}{cc}e_{0} & 0 \\ 0 & e_{1}\end{array}\right)$, then by Proposition 6.5 the plane $P$ contains $e_{0}$ and $e_{1}$. Furthermore any line in $P$ though one of these points is either contained in $S$ or intersects $S$ in a scheme of length at least 6 . Since $P \cap S$ contains a plane quintic $C$ but no plane sextic $e_{0}$ and $e_{1}$ must be residual to $C$.

If $B_{1}=\left(\begin{array}{cc}e_{1} & 0 \\ e_{0} & e_{1}\end{array}\right)$, then by Proposition 6.5 the point $e_{1}$ is residual to $C$ in $P$. There are two cases

(1) $e_{0} \in P$. Then $B_{1}$ vanishes on every $\mathrm{P}^{3}$ containing $P$. As in the proof of Proposition 6.20 the general residual space curve $D$ must be of degree 6 and genus 2. This implies that the residual scheme in $P$ has length 2 .

(2) $e_{0} \notin P$. In this case we can assume $P=e_{1} \wedge e_{2} \wedge e_{3}$ and

$$
B=\left(\begin{array}{ccc}
e_{3} \wedge e_{2} & e_{1} & e_{0} \\
e_{3} \wedge e_{2}+e_{1} \wedge * & 0 & e_{1}
\end{array}\right)^{T}
$$


by Proposition 6.5. Furthermore $B$ must have a syzygy $a=\left(e_{1}, q_{1}, q_{2}\right)$ with $q_{1}$ and $q_{2}$ forms of degree 2 . Equivalently

$$
B^{\prime}=\left(\begin{array}{ccc}
e_{3} \wedge e_{2} \wedge e_{1} & e_{1} & e_{0} \\
e_{3} \wedge e_{2} \wedge e_{1} & 0 & e_{1}
\end{array}\right)^{T}
$$

must have a syzygy $\left(1, q_{1}, q_{2}\right)$ which is impossible since the first row of $B^{\prime}$ is independent of the last two.

In both case we therefore have a length 2 scheme $R$ residual to $C$ in $P$. Therefore $S$ is not cut out by sextics and $b=1$.

Remark 6.23. If $a=1$ and $b=1$, then $L=\mathrm{P}_{\text {span }}$ is a line in $P$ that contains a length two subscheme residual to $C$. The line $L$ is either a 7-secant or $L \subset S$ with $L^{2} \leq-5$.

We may summarize our results

Corollary 6.24. Any smooth surfaces in $\mathrm{P}^{4}$ with Hilbert polynomial $P_{S}(n)=11 / 2 n^{2}-9 / 2 n+1$ is rational and has one of three possible Hilbert functions distinguished by their B matrices. They differ from the Hilbert polynomial only in the degrees $n=1, \ldots, 5$ where they take the values

(1) $(5,15,35,70,116)$ and $B=\left(e_{3} \wedge e_{2}, e_{1}, e_{0}\right)^{T}, B=\left(e_{2} \wedge e_{1}, e_{0}, 0\right)^{T}$, or $B=\left(0, e_{1}, e_{0}\right)^{T}$;

(2) $(5,15,35,69,116)$ and $B=\left(\begin{array}{lll}q_{1} & e_{0} & e_{3} \\ q_{2} & e_{1} & e_{4}\end{array}\right)^{T}$;

(3) $(5,15,35,69,115)$ and $B=\left(\begin{array}{lll}q_{1} & e_{0} & 0 \\ q_{2} & 0 & e_{1}\end{array}\right)^{T}$ or $B=\left(\begin{array}{lll}q_{1} & e_{1} & e_{0} \\ q_{2} & 0 & e_{1}\end{array}\right)^{T}$.

Proof. First, any smooth surface with this Hilbert polynomial has degree 11, sectional genus $11, K_{S}^{2}=-11$ and Euler characteristic $\chi_{S}=1$. Such a surface is nonrational if only if $2 K_{S}$ is effective, by Castelnuovo's criterion. But since any minimal surface has $K_{S}^{2} \geq 0$, any curve in $\left|2 K_{S}\right|$ has at least 11 exceptional -1-curves with multiplicity 2 as components. Now, $H \cdot K_{S}=9$ so this is clearly impossible, and we conclude that any smooth surface with the given Hilbert polynomial is rational.

The Hilbert function $H_{S}(n)$ is, of course, computed by $h^{0}\left(\mathscr{O}_{\mathrm{P}^{4}}(n)-\right.$ $h^{0}\left(\mathscr{I}_{S}(n)\right)$ so the difference to the Hilbert polynomial is the dimensions of the higher cohomology groups of $\mathscr{I}_{S}(n)$. The different possibilities for these groups are determined in Propositions 6.1, 6.13, 6.14 and Corollary 6.17, and correspond to the following values of $a=h^{1}\left(\mathscr{I}_{S}(4)\right)-1$ and $b=h^{1}\left(\mathscr{I}_{S}(5)\right)$, namely $a=b=0, a=1$ and $b=0$, and $a=b=1$. The values of $h^{0}\left(\mathscr{I}_{S}(n)\right)$ and consequently of the Hilbert function then follows from the diagram of Proposition 6.1. 
Surfaces with the first Hilbert function were treated by Popescu in [14]. Examples of surfaces with the second Hilbert function were found by v. Bothmer, Erdenberger and Ludwig [16]. We treat this case in more detail in the next sections, both giving two different new constructions of such surfaces, and showing that they belong to a unique irreducible and unirational family. In the third case there are irreducible surfaces with this Hilbert function that are singular along $\mathrm{P}_{\text {span. }}$. We have not found any smooth surface with this Hilbert function, but also cannot rule it out.

\section{Rational Surfaces with $d=11, \pi=11, a=1, b=0$}

Recall that, in the notation of the previous section, when $a=1$ and $b=0$ we have that $B_{1}$ is a $2 \times 2$ matrix and its entries span a $\mathrm{P}^{3}$.

Proposition 7.1. Let $\mathrm{P}_{\text {span }}=\mathrm{P}^{3}$ and consider the smooth quadric $Q:=$

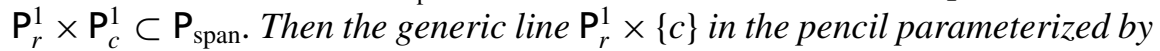
the columns of $B_{1}$ intersects $S$ in a scheme of length at least 5 and the special plane $P$ in one point. Furthermore $D^{2}<2$.

Proof. Since $Q$ can not be contained in $S$ the generic line must intersect $S$ in a scheme of finite length. By Proposition 6.5 this length is at least 5. Since $Q$ is smooth, $P$ can not be contained in $Q$ and the intersection of the generic line with $P$ must be proper. Finally, $D^{2} \leq 2$ by Lemma 6.3 . But equality here means that $S$ intersects $P$ in a scheme of length two residual to $C$, so $\mathrm{S}$ is not cut out by sextics and $b>0$.

We denote $C_{S P}, C_{S Q}$ and $C_{P Q}$ the curve components of $S \cap P, S \cap Q$ and $P \cap Q$ respectively. Also we set $H=\mathrm{P}_{\text {span }} \cap S$ and $E=H-C_{S Q}$.

Proposition 7.2. $C_{S Q}$ is of type $(5,3)$ on $Q$ and $P$ intersects $Q$ in a line of type $(1,0)$.

Proof. Since $B_{1}$ vanishes on $\mathrm{P}_{\text {span }}$ the curve $H=S \cap \mathrm{P}_{\text {span }}$ lies on 3 quartics and no cubic by Proposition 2.5. Since the general line in one of the rulings of $Q$ is at least a 5-secant all quartics must contain $Q$. Therefore the residual curve $E=H-C_{S Q}$ lies on exactly 3 quadrics and no cubic. By Lemma 6.19 we obtain that $E$ is determinantal of degree 3 and arithmetic genus 0 . Consequently $C_{S Q}$ has degree $8=11-3$. Now the number of quintics independent of the quartics is at least

$h^{0}\left(\mathcal{O}_{\mathrm{P}^{3}}(5)\right)-h^{0}\left(\mathscr{O}_{C_{S Q}}(5)\right)-3 \cdot 4+s=56-(55+1-11)-12+s=s-1$

where $s$ is the number of linear syzygies between the quartics. Since the ideal of $D$ has 2 linear syzygies between the 3 quadrics, so do the three quartics 
obtained by multiplying with $Q$. Therefore the ideal of $H$ contains at least one independent quintic. This shows that $C_{S Q}$ is contained in a divisor of type $(5,5)$. So $C_{S Q}$ is of type $(5,3)$ or $(4,4)$. Since every line of type $(0,1)$ on $Q$ is at least a 5-secant, the second choice is not possible.

Now consider the intersection $P \cap \mathrm{P}_{\text {span }}$. If $P$ is a subset of $\mathrm{P}_{\text {span }}, H$ must contain a plane quintic. Since $Q$ is smooth, $Q \cap P$ must be a plane conic in this case and consequently $E$ a plane cubic. This contradicts Lemma 6.19. So $L=P \cap \mathrm{P}_{\text {span }}$ is a line. By Proposition 6.5 it must intersect all 5-secants on $Q$. Since the general divisor of type $(0,1)$ must be such a 5 -secant by the arguments above, we obtain that $L$ is of type $(1,0)$.

Recall that reducible surface is called Zappatic if its components and the pairwise intersections of two components are smooth. [3].

Proposition 7.3. The union $S \cup Q \cup P$ is linked $(4,5)$ to a surface $B$ of degree $d=6$ sectional genus $\pi=3$. If $S \cup Q \cup P$ is Zappatic, then $B$ is locally Cohen-Macaulay with $\chi_{B}=1$.

Proof. First we note that any quintic hypersurface that contains $S \cup P$ also contains $Q$. The reason is simply that the intersection $(S \cup P) \cap Q=C_{S Q} \cup C_{P Q}$ is a curve of type $(6,3)$ on the quadric. Since the intersection $C_{S P}$ is a quintic curve, the space of quintics in the ideal of $S$ that contains $S \cup P \cup Q$ has codimension at most one in the space of all quintics in this ideal. Since $S$ lies on a unique quartic and on 10 quintics, this means that there is at least a 4-dimensional space of quintics that contains $S \cup P \cup Q$ independent of the quartic.

Thus in the intersection of the unique quartic and a general quintic in its ideal, the surface $S \cup P \cup Q$ is linked to a surface $B$ of degree 6 .

The arithmetic genus $\pi_{U}$ of the union $U=S \cup P \cup Q$ is computed by the formula

$$
\begin{aligned}
\pi_{U} & =\pi_{S}+\pi_{P}+\pi_{Q}+\operatorname{deg}\left(C_{S P}\right)+\operatorname{deg}\left(C_{S Q}\right)+\operatorname{deg}\left(C_{P Q}\right)-2 \\
& =11+5+8+1-2=23
\end{aligned}
$$

The arithmetic genus $\pi_{B}$ of $B$ is computed by the formula for liaison [15] of space curves:

$$
\pi_{U}-\pi_{B}=\frac{4+5-4}{2}(\operatorname{deg}(U)-\operatorname{deg}(B))=\frac{5 \cdot(14-6)}{2}=20,
$$

so $\pi_{B}=3$.

Now assume that $U$ is Zappatic. In particular we assume that the three curves $C_{S P}, C_{S Q}$ and $C_{P Q}$ are smooth. Furthermore we assume that the intersection $S \cap P \cap Q$ consists of 5 distinct points on the line of intersection between $P$ 
and $Q$. In three of these points all three components meet pairwise in a curve, so the tangent cone of the union is three planes that meet pairwise in a line. These points are Zappatic singularities of type $E_{3}$ in the notation of [3].

In the remaining two points, the isolated intersection points of $S \cap Q$, the plane $P$ intersect both $S$ and $Q$ along a curve. Therefore the tangent cone to the union of the three surfaces at each of these points is the cone over three lines that form a cubic space curve of arithmetic genus 0 . So these points are Zappatic singularities of type $R_{3}$. Let $f=3$ be the number of $E_{3}$ singularities of the Zappatic surface $U$. Then

$$
\begin{aligned}
\chi_{U} & =\chi_{S}+\chi_{Q}+\chi_{P}-\chi_{C_{S P}}-\chi\left(C_{S Q}\right)-\chi\left(C_{P Q}\right)+f \\
& =1+1+1+5+7-1+3=17
\end{aligned}
$$

by the formula (3.16) of [3]. Now we are in a position to compute $\chi_{B}$. We already computed $d_{B}=6$ and $\pi_{B}=3$, so $\chi(\mathscr{O}(d H))=3 d^{2}+d+\chi_{B}$. By the liaison exact sequence

$$
0 \longrightarrow \omega_{U} \longrightarrow \omega_{U \cup B} \longrightarrow \mathscr{O}_{B}(4) \longrightarrow 0
$$

we have $\chi_{U \cup B}-\chi_{U}=3 \cdot 4^{2}+4+\chi_{B}$. But $U \cup B$ is a complete intersection of type $(4,5)$ and therefore has $\chi_{U \cup B}=70$. Thus $\chi_{B}=1$.

Finally a Zappatic surface is locally Cohen-Macaulay so by linkage $B$ is also locally Cohen-Macaulay.

\section{Counting dimensions}

Proposition 8.1. Let $S \subset \mathrm{P}^{4}$ be a smooth surface of degree $d$, sectional genus $\pi$ and Euler characteristic $\chi_{S}$ and $\mathcal{N}$ the normal bundle on $S$ in $\mathrm{P}^{4}$. Then

$$
\chi(\mathcal{N})=d(10-d)+5(\pi-1)+2 \chi_{S}
$$

Proof. By Hirzebruch-Riemann-Roch we have

$$
\chi(\mathcal{N})=\frac{1}{2}\left(c_{1}(\mathcal{N})^{2}-2 c_{2}(\mathcal{N})\right)-\frac{1}{2} c_{1}(\mathcal{N}) K_{S}+2 \chi_{S}
$$

on a surface. Substituting $c_{1}(\mathcal{N})=5 H+K$ and $c_{2}(\mathcal{N})=d^{2}$ and applying the adjunction formula $2 \pi-2=H(H-K)$, we obtain the formula above.

Remark 8.2. Since $h^{2}(\mathcal{N})=0$ for a rational surface, $\chi(\mathcal{N})$ is the expected dimension of the component of the Hilbert scheme on which $S$ lies. [12]. In case $S$ is rational of degree 11 and sectional genus 11, we obtain

$$
\chi(\mathcal{N})=-11+5 \cdot 10+2 \cdot 1=41 .
$$


In the previous sections we analyzed smooth rational surfaces of degree 11 and sectional genus 11 that lie on a quartic hypersurface and whose ideal is generated in degree 6 , i.e. has Hilbert function $H_{S}(n)=\{1,5,15,35,69$, $\left.11 / 2 n^{2}-9 / 2 n+1\right\}$. More precisely we determined the possible linear parts of the maps of the Tate resolution $T\left(\mathscr{I}_{S}\right)$

$$
\cdots \longrightarrow 3 E(-1) \stackrel{\alpha}{\longrightarrow} E(-2) \oplus 2 E(-3) \oplus E(-4) \stackrel{\beta}{\longrightarrow} 2 E(-4) \longrightarrow \cdots
$$

of such surfaces.

The entries of these maps are forms on $\left(\mathrm{P}^{4}\right)^{*}$, so the pure forms correspond to linear subspaces of $\mathrm{P}^{4}$. In particular, the entries of the linear parts of the maps are points in $\mathrm{P}^{4}$. We determined that the linear part of $\alpha$ has three entries that span a plane $P=\mathrm{P}^{2}$, while the linear part of $\beta$ is a $2 \times 2$-matrix whose entries span $\mathrm{P}_{\text {span }}=\mathrm{P}^{3}$ and where the columns (and rows) span the vertical (and horizontal lines) of a smooth quadric surface $Q$. In this notation we count the parameters for the corresponding Tate resolutions and find that they coincide with the expected dimension.

Proposition 8.3. The family $F$ of minimal complexes

$$
3 E(-1) \stackrel{\alpha}{\longrightarrow} E(-2) \oplus 2 E(-3) \oplus E(-4) \stackrel{\beta}{\longrightarrow} 2 E(-4)
$$

such that the linear part of $\beta$ determine a smooth quadric surface $Q \subset \mathrm{P}_{\text {span }}=$ $\mathrm{P}^{3}$ and the linear part of $\alpha$ spans $P=\mathrm{P}^{2}$ with $P \cap Q$ a line defined by a row of $B_{1}$, is irreducible, rational and of dimension 41 .

Proof. First we can choose a plane $P \subset \mathrm{P}^{4}$ and a smooth quadratic surface $Q \subset \mathrm{P}^{3} \subset \mathrm{P}^{4}$ such that $L=P \cap \mathrm{P}^{3}=P \cap Q$ is a line. For each such choice we can represent $\alpha$ and $\beta$ by matrices of the form

$$
A=\left(\begin{array}{cccc}
e_{0} & q_{1} & q_{4} & r_{1} \\
e_{1} & q_{2} & q_{5} & r_{2} \\
e_{2} & q_{3} & q_{6} & r_{3}
\end{array}\right) \quad B=\left(\begin{array}{cc}
p_{1} & p_{2} \\
e_{0} & e_{1} \\
e_{3} & e_{4} \\
0 & 0
\end{array}\right)
$$

where $P=\left\langle e_{0}, e_{1}, e_{2}\right\rangle, L=\left\langle e_{0}, e_{1}\right\rangle$ and $\mathrm{P}_{\text {span }}=\left\langle e_{0}, e_{1}, e_{3}, e_{4}\right\rangle$. The entries $p_{i}$ and $q_{i}$ are 2-forms and $r_{i}$ are 3 -forms. Notice that the relation $A B=0$ is linear in the coefficients of the 2-forms. In the corresponding linear system, we have 80 coefficients of 2-forms in $A$ and $B$ and six 3-forms with 10 coefficients in $A B$, so we expect a 20-dimensional solution. But in fact the relations of $A B=$ 0 are dependent and we find a 25 -dimensional affine solution space. See [17] for the calculation. Now the quadratic part of both matrices is only defined modulo the linear part. Projectively we obtain a $14=25-2 \cdot 5-1$ dimensional solution space. The cubics can be chosen arbitrarily, but are defined only modulo the 
linear and quadratic part of $A$. Furthermore the two syzygies of $A$ given by $B$ are degree 3 dependencies between the linear and the quadratic part, so we obtain a $11=30-1 \cdot 10-2 \cdot 5-1+2$ dimensional space of possible degree 3 parts of $A$. In total we have shown that the family of complexes $F$ is birationally parameterized by an irreducible and Zariski open set

$$
X \subset \mathrm{G}(2,5) \times\left(\mathrm{P}^{4}\right)^{*} \times \mathrm{P}_{\text {quadrics }}^{6} \times \mathrm{P}_{\text {deg2part }}^{14} \times \mathrm{P}_{\text {deg } 3 \text { part }}^{11} .
$$

In particular $\operatorname{dim} X=6+4+6+14+11=41$.

THEOREM 8.4. The family of smooth rational surfaces of degree 11, sectional genus 11 with Hilbert function $H_{S}(n)=\left\{1,5,15,35,69,11 / 2 n^{2}-\right.$ $9 / 2 n+1\}$ is unirational, irreducible of dimension 41 . The general member of the family is linked $(4,5)$ to a Zappatic surface $P \cup Q \cup B$, where $P$ is a plane, $Q$ is a smooth quadric surface and $B$ is a smooth Bordiga surface, such that $L=P \cap Q$ is a line, $B \cap Q$ consists of three distinct lines that intersect $L$, and $P \cap B$ is the union of a line distinct from $L$ and two points on $L$. In particular $S \cup P \cup Q$ is an arithmetically Cohen-Macaulay surface defined by the $4 \times 4$ minors of a $4 \times 5$ matrix with 4 columns of linear forms and 1 column of quadratic forms.

Proof. By the previous proposition it remains for the first part to give an example. This is done by choosing random matrices $A$ and $B$ satisfying the above conditions, computing the minimal free resolution of $\mathscr{I}_{S}$ via the BGGCorrespondence and checking that this is an ideal sheaf defining a smooth surface by the Jacobian criterion. An effective procedure is to compute an example over a finite characteristic. This is done with with Macaulay 2 and documented at [17]. The fact that the general member is linked $(4,5)$ to a Zappatic surface is an open condition that is also checked in an example. Finally, since a Bordiga surface is an arithmetically Cohen-Macaulay surface defined by the $3 \times 3$ minors of a $3 \times 4$ matrix with linear entries, the linked surface $S \cup P \cup Q$ is also arithmetically Cohen-Macaulay, and the proposition follows.

REMARK 8.5. The example by v. Bothmer, Erdenberger and Ludwig is a blowup of the plane in 20 points. In fact the linear system has the form $9 L-$ $3 E_{1}-\sum_{i=2}^{15} 2 E_{i}-\sum_{i=16}^{20} E_{i}$ where $L$ is the pullback of a line from the plane, while the $E_{i}, i=1, \ldots, 20$ are the exceptional divisors of the blowup. From Theorem 8.4 it follows that the linear system of any smooth surface with the second Hilbert function has this form. The challenge remains to determine necessary and sufficient conditions for position of the 20 points in $\mathrm{P}^{2}$. 


\section{Construction}

We can use the properties of a surface $S$ as in the Theorem 8.4 to give a geometric construction

Construction 9.1.

(1) Choose a line $L$ in $\mathrm{P}^{2}$

(2) Choose general points $P_{1}, P_{2}$ and $P_{3}$ on $L$

(3) Choose general points $P_{4}, \ldots, P_{8}$ outside of $L$

(4) Let $C$ be the unique irreducible quartic curve that contains $P_{1}, \ldots, P_{8}$ and is singular in $P_{3}, P_{4}$ and $P_{5}$

(5) Choose general points $P_{9}$ and $P_{10}$ on $C$

(6) Let $B$ be the blowup of $\mathrm{P}^{2}$ in $P_{1}, \ldots, P_{10}$ and denote the exceptional divisors by $E_{1}, \ldots, E_{10}$.

(7) Embed $B$ in $\mathrm{P}^{4}$ with the linear system $|H|:=\left|4 L-E_{1}-\ldots-E_{10}\right|$ the image will be a Bordiga surface of degree 6 and sectional genus 3

(8) Let $\mathrm{P}_{\text {span }}$ be the hyperplane in $\mathrm{P}^{4}$ corresponding to $C \in|H|$. Since $C$ is singular in $P_{3}, P_{4}$ and $P_{5}, \mathrm{P}_{\text {span }}$ contains the exceptional lines $E_{3}, E_{4}$ and $E_{5}$.

(9) Let $Q \subset \mathrm{P}_{C}^{3}$ be the unique quadric containing these lines.

(10) Let $\widetilde{L}$ be the strict transform of $L$. It is again a line in $\mathrm{P}^{4}$. Let $P$ be the unique $\mathrm{P}^{2}$ containing $\widetilde{L}$ and intersecting $E_{4}$ and $E_{5}$.

(11) Let $S$ be a $(4,5)$ linkage of $P \cup Q \cup B$.

THEOREM 9.2. The construction 9.1 yields a 41-dimensional unirational, irreducible family of smooth rational surfaces $S$ of degree 11 and sectional genus 11 with precisely two 6-secants lying on a unique quartic hypersurface.

Proof. It is straightforward to check that each step of the construction is possible except the last one. For the last step we need to show that $P \cup Q \cup B$ lies on a quartic and a quintic hypersurface with no common component. For this we first consider the exact sequence of ideal sheaves

$$
\left.0 \longrightarrow I_{B}(2) \longrightarrow I_{B \cup Q}(3) \longrightarrow I_{B \cup Q}\right|_{H}(3) \longrightarrow 0
$$

where the first map is multiplication by the linear form defining the hyperplane $H$ that contains $Q$. All cohomology groups on the left and on the right vanish: On the left $B$ is arithmetically Cohen-Macaulay and does not lie on any quadric, while on the right $\left.I_{B \cup Q}\right|_{H}$ is the ideal sheaf in $\mathscr{O}_{H}$ of the union of $Q$ and the twisted cubic curve $C$ on $B \cap H$ residual to $Q$. Therefore the cohomology of $\left.I_{B \cup Q}\right|_{H}(3)$ coincides with the cohomology of $\left.I_{C}\right|_{H}(1)$, which vanishes. We 
conclude that the cohomology groups of the sheaf in the middle also vanishes. Similarly, twisting with $\mathscr{O}_{\mathrm{P}^{4}}(1)$, we get $h^{0}\left(I_{B \cup Q}(4)\right)=7$ and $h^{1}\left(I_{B \cup Q}(4)\right)=0$. Next, consider the exact sequence of ideal sheaves

$$
\left.0 \longrightarrow I_{B \cup Q}(3) \longrightarrow I_{B \cup Q \cup P}(4) \longrightarrow I_{B \cup Q \cup P}\right|_{H^{\prime}}(4) \longrightarrow 0
$$

where the first map is the multiplication by the linear form defining a general hyperplane $H^{\prime}$ through $P$. By the above, $B \cup Q \cup P$ lies in a quartic hypersurface if and only if $(B \cup Q \cup P) \cap H^{\prime}$ lies on a quartic surface in $H^{\prime}$. But $(B \cup Q \cup$ $P) \cap H^{\prime}$ is the union $P \cup E \cup L^{\prime}$, of the plane $P$, an elliptic quintic curve $E$ in $H^{\prime} \cap B$ with a trisecant line $L$ in $P$ and a line $L^{\prime}$ such that $L \cup L^{\prime}=Q \cap H^{\prime}$. So $(B \cup Q \cup P) \cap H^{\prime}$ is contained in a quartic surface if and only if $E \cup L^{\prime}$ is contained in a cubic surface. Since $E \cup L=H^{\prime} \cap B$ lies in 4 cubics, and $L^{\prime}$ meets $L$, there is at least one cubic surface that contains $E \cup L^{\prime}$, and hence at least one quartic hypersurface that contains $B \cup Q \cup P$.

On the other hand, there are 15 quartics that contain $E$, i.e. 10 quartics that contain $E \cup L^{\prime}$. Hence, as above,

$$
h^{0}\left(I_{B \cup Q \cup P}(5)\right)=h^{0}\left(I_{B \cup Q}(4)\right)+h^{0}\left(\left.I_{B \cup Q \cup P}\right|_{H^{\prime}}(5)\right)=7+10=17
$$

and $B \cup Q \cup P$ lies in 12 quintic hypersurfaces that are independent of the quartic. Since the Bordiga surface is not contained in any reducible cubic hypersurface, $B \cup Q$ is not contained in any cubic hypersurface and $Q \cup P$ is not contained in any hyperplane, any quartic that contains $B \cup Q \cup P$ must be irreducible. Therefore the general quintic and quartic that contains $B \cup Q \cup B$ have no common component and so $B \cup Q \cup B$ is linked $(4,5)$ to a surface $S$.

The parameters involved in the construction form an open set in

$$
Y \subset\left(\mathrm{P}^{2}\right)^{*} \times\left(\mathrm{P}^{1}\right)^{3} \times\left(\mathrm{P}^{2}\right)^{5} \times(C)^{2} \times \operatorname{Aut}\left(\mathrm{P}^{4}\right) \times \mathrm{P}_{\text {linkage }}^{11} .
$$

Since $C$ is rational this proves that our family is unirational. To find the dimension of the Hilbert scheme component we need to subtract dimension of the automorphisms of $\mathrm{P}^{2}$ (projective dimension 8 ) and the dimension of the space of independent quintic hypersurfaces that contain $S \cup P \cup Q$ (projective dimension 3 by Theorem 8.4), i.e. the space of Bordiga surfaces that lead to the same $S$. Therefore these surfaces $S$ belong to a

$$
2+3+10+2+24+11-8-3=41
$$

dimensional family in the Hilbert scheme.

Next we compute the Hilbert polynomial of $S$.

Of course, the degree of $S$ is 11 . The sectional genus is given by the liaison formula

$$
\pi_{S}-\pi_{B \cup P \cup Q}=5 / 2(11-9)=5
$$


i.e. $\pi_{S}=11$ since the sectional genus of $B \cup Q \cup P$ is 6 . To get the Euler characteristic $\chi_{S}$ we first compute $\chi_{B \cup Q \cup P}$. By construction $B \cup Q \cup P$ is Zappatic, i.e. the three components $B, P, Q$ and their pairwise intersections are smooth. Furthermore the intersection $B \cap Q \cap P$ consists of precisely three points on the line $L=P \cap Q$. At one of them, the point $L \cap L^{\prime}$, the three surface component intersect pairwise in codimension 1 , so this point is a Zappatic singularity of type $E_{3}$ on the union. At the two other points, $B$ and $P$ intersect in codimension 2, while the $B$ and $Q$ and $Q$ and $P$ intersect in codimension 1, so these points are Zappatic singularities of type $R_{3}$. The Euler characteristic of $B \cup Q \cup P$ is therefore

$$
\begin{aligned}
\chi_{B \cup Q \cup P} & =\chi_{B}+\chi_{Q}+\chi_{P}-\chi_{L^{\prime}}-\chi_{L}-\chi_{B \cap Q}+f \\
& =1+1+1-1-1-3+f=f-2
\end{aligned}
$$

by the formula for Zappatic surfaces. Since $f=1$ count the number of Zappatic singularities of type $E_{3}$, we get $\chi_{B \cup Q \cup P}=-1$. In the liaison exact sequence

$$
0 \rightarrow \omega_{B \cup Q \cup P} \rightarrow \omega_{(4,5)} \rightarrow \mathscr{O}_{S}(4) \rightarrow 0
$$

The Euler characteristic of the first two sheaves are -1 and 70 respectively, while the Hilbert polynomial of $\mathscr{O}_{S}$ is $P_{S}(d)=11 / 2 d^{2}-9 / 2 d+\chi_{S}$. In particular $P_{S}(4)=70+\chi_{S}$, so by the exact sequence $\chi_{S}=1$.

To see that the general surface of this family is of the kind found in the previous section, we have checked one example for smoothness (see [17]). Since Popescu [14] showed that there are no nonrational surfaces of these invariants, $S$ must be rational (cf. also the proof of Corollary 6.24). Furthermore, by liaison, $S \cap Q$ is a curve of type $(3,5)$, while $L$ is of type $(0,1)$. So two of the three lines in $B \cap Q$ are 6-secants to $S$.

\section{REFERENCES}

1. Bê̆linson, A. A., Coherent sheaves on $\mathbf{P}^{n}$ and problems in linear algebra, Funktsional. Anal. i Prilozhen. 12(3) (1978), 68-69.

2. Bernštěn, I. N., Gel'fand, I. M., and Gel'fand, S. I., Algebraic vector bundles on $\mathbf{P}^{n}$ and problems of linear algebra, Funktsional. Anal. i Prilozhen. 12(3) (1978), 66-67,

3. Calabri, Alberto, Ciliberto, Ciro, Flamini, Flaminio, and Miranda, Rick, On the geometric genus of reducible surfaces and degenerations of surfaces to unions of planes, In The Fano Conference, pages 277-312. Univ. Torino, Turin, 2004.

4. Decker, Wolfram, Ein, Lawrence, and Schreyer, Frank-Olaf, Construction of surfaces in $\mathbf{P}^{4}$, J. Algebraic Geom. 2(2) (1993), 185-237.

5. Eisenbud, David, Fløystad, Gunnar, and Schreyer, Frank-Olaf, Sheaf cohomology and free resolutions over exterior algebras, Trans. Amer. Math. Soc. 355(11) (2003), 4397-4426 (electronic).

6. Ellingsrud, Geir, and Peskine, Christian, Sur les surfaces lisses de $\mathbf{P}^{4}$, Invent. Math. 95(1) (1989), 1-11. 
7. Eisenbud, David, Schreyer, Frank-Olaf, and Weyman, Jerzy, Resultants and Chow forms via exterior syzygies, J. Amer. Math. Soc. 16(3) (2003), 537-579 (electronic).

8. Fulton, W., and Harris, J., Representation Theory, Grad. Texts in Math. 129 (1991).

9. Fulton, William, Intersection theory, volume 2 of Ergeb. Math. Grenzgeb. 3. Folge. A Series of Modern Surveys in Mathematics [Results in Mathematics and Related Areas. 3rd Series. A Series of Modern Surveys in Mathematics]. second edition, 1998.

10. Gruson, Laurent, and Peskine, Christian, Genre des courbes de l'espace projectif, II, Ann. Sci. École Norm. Sup. (4) 15(3) (1982), 401-418.

11. Green, Mark L., Generic initial ideals, In Six lectures on commutative algebra (Bellaterra, 1996), Progr. Math. 166 (1998), 119-186.

12. Grothendieck, Alexander, Techniques de construction et théorèmes d'existence en géométrie algébrique, IV, Les schémas de Hilbert, In Séminaire Bourbaki, Vol. 6, pages Exp. No. 221, 249-276. Soc. Math. France, Paris, 1995.

13. Grayson, Daniel R., and Stillman, Michael E., Macaulay 2, a software system for research in algebraic geometry, Available at http://www.math.uiuc.edu/Macaulay2, 2002.

14. Popescu, Sorin, Surfaces of degree $\geq 11$ in the Projective Fourspace, Dissertation, Universität des Saarlandes, 1993.

15. Peskine, C., and Szpiro, L., Liaison des variétés algébriques, I, Invent. Math. 26 (1974), 271-302.

16. v. Bothmer, H.-Chr. Graf, Erdenberger, C., and Ludwig, K., A new family of rational surfaces in $\mathrm{P}^{4}$, J. Symbolic Comput. 29(1) (2005), 51-60.

17. v. Bothmer, H.-Chr. Graf, and Ranestad, K., Macaulay 2 scripts for constructing rational surfaces of degree 11 and sectional genus 11 in $\mathrm{P}^{4}$, Available at http://www-ifm.math.unihannover.de//bothmer/degree11. See also the LaTeX-file of this article at http://arXiv.org., arXiv:math/0603567v2, 2006.

INSTITIUT FÜR ALGEBRAISCHE GEOMETRIE LEIBNITZ UNIVERSITÄT HANNOVER

WELFENGARTEN 1

D-30167 HANNOVER

GERMANY

URL: http://www-ifm.math.uni-hannover.de/ 'bothmer
MATEMATISK INSTITUTT

POSTBOKS 1053,

BLINDERN

NO-0316 OSLO

NORWAY

URL: http://folk.uio.no/ranestad/ 